\title{
Geometry of the String Equations
}

\author{
Gregory Moore* \\ Department of Physics, Yale University, New Haven, CT 06511-8167, USA
}

Received May 11, 1990

\begin{abstract}
The string equations of hermitian and unitary matrix models of $2 D$ gravity are flatness conditions. These flatness conditions may be interpreted as the consistency conditions for isomonodromic deformation of an equation with an irregular singularity. In particular, the partition function of the matrix model is shown to be the tau function for isomonodromic deformation. The physical parameters defining the string equation are interpreted as moduli of meromorphic gauge fields, and the compatibility conditions can be interpreted as defining a "quantum" analog of a Riemann surface. In the latter interpretation, the equations may be viewed as compatibility conditions for transport on "quantum moduli space" of correlation functions in a theory of free fermions. We discuss how the free fermion field theory may be deduced directly from the matrix model integral. As an application of our formalism we discuss some properties of the BMP solutions of the string equations. We also mention briefly a possible connection to twistor theory.
\end{abstract}

\section{Introduction and Conclusion}

Recently there has been some remarkable progress in the theory of $2 D$ gravity and string theory [1-6]. The basic equations governing nonperturbative $2 D$ gravity coupled to minimal models have been discovered. An exciting feature of these equations is their close relation with the KP hierarchy, indicating the existence of some interesting underlying mathematical structure. While the connection to the KP hierarchy per se is likely to be peculiar to the minimal models, one may hope that a thorough examination of these systems will lead to the discovery of structures applicable to general models of $2 D$ gravity. In this paper we attempt to construct a mathematical framework for the string equations in the hope that some qualitative features of this framework will persist in the general case.

* (moore @yalphy.hepnet, or @yalehep.bitnet) 
We would like some geometrical interpretation of the string equations. One very interesting interpretation is provided by Witten's theory of topological gravity [7-10]. In the present paper we suggest another route which follows more closely the wellestablished paradigm for the geometry of conformal field theory. Recall the main elements of Friedan and Shenker's "modular geometry" [11]. The conformal blocks of a correlation function are horizontal sections of a flat vector bundle over the moduli space of curves. A horizontal section satisfies a differential equation which essentially follows from the idea that the stress energy tensor defines a connection on the bundle. If we discuss nontrivial (nonrational) conformal field theories, e.g., those associated with nonlinear sigma models with Calabi-Yau spaces as targets, the flatness of the connection is the condition that the spacetime equations of motion are satisfied, i.e., that the appropriate generalizations of Einstein's equations are satisfied.

We propose that a similar picture holds in the case of $2 D$ gravity. We begin by writing the string equations as flatness conditions. These conditions are compatibility conditions for transport equations in a space parametrized by $x, T_{j}$, the cosmological constant and the masses associated to the $2 D$ gravity model. The parameters $x, T_{j}$, together with the initial conditions for the nonlinear differential equations known as the "string equations," are identified with the moduli of a certain class of meromorphic gauge fields on $\mathbb{P}^{1}$. This moduli space is given a further interpretation in Sect. 5 as a generalization of the moduli space of curves. The analogy to conformal field theory is developed further in Sect.6, where we interpret the transport equations in $x, T_{j}$ as Knizhnik-Zamalodchikov-type equations for a free fermion construction of current algebra. The novel element is that the correlators in question involve operators (dubbed "star operators") which are not normally considered in conformal field theory. In Sect. 7 we suggest how one might establish a direct connection between the formalism of this paper and the random matrix formulation of $2 D$ gravity.

It is well-known that the quantum field theory of free fermions on a curve provides an elegant framework for understanding much of the theory of the quasiperiodic solutions of the generalized $\mathrm{KdV}$ hierarchies. Following some observations of Gross and Migdal [3], Douglas emphasized the importance of the generalized $\mathrm{KdV}$ hierarchies in [5]. This led to the suggestion [5,6] that the partition function of the matrix model might be a tau function in the sense of $[12,13]$. While not strictly true, we show that this conjecture is essentially correct: the partition function of $2 D$ gravity is given by the tau function for an isomonodromic deformation problem closely related to that of the stationary $\mathrm{KdV}$ equations. The tau function in the quasiperiodic case admits an interesting interpretation as a function on an orbit of a loop group [12,14], and it would be very interesting to find an analogous interpretation in this case.

In an effort to demonstrate that the above picture is not merely useless reinterpretation of know results we have shown in Appendix A how the present formalism can be used to establish some properties of the string equations which have recently become interesting in connection with the so-called "nonperturbative violation of universality" in matrix models.

It has been repeatedly emphasized by Atiyah, Hitchin, Ward, and Witten that low-dimensional integrable differential equations and field theories should be related to higher dimensional gauge theory. The four-dimensional self-dual Yang-Mills equations are expected to play a central role in such a formulation. 
In Appendix B we sketch some connections between those ideas and the ideas of this paper.

After we completed most of this work we found that some ideas similar to those of Sects. 2, 3, and 5, in the context of the MKdV hierarchy and the associated PII equation, have been discussed by Flaschka and Newell [15]. V. Korepin also pointed out to us some overlap between the remarks of Sect. 7 and those of [16]. We have been informed by E. Martinec of similar progress, especially in relating the gravity partition function to a tau function [17].

\section{String Equations as Flatness Conditions}

Let us recall how M. Douglas wrote the general $(p, q)$ string equations in [5]. If $L=D^{q}+u_{q-2} D^{q-2}+\cdots+u_{0}$ is the continuum limit of a multiplication operator $f(\lambda) \rightarrow \lambda f(\lambda)$ on the orthogonal polynomials $f$ in a matrix chain model then, he argued, the continuum limit of the conjugate derivative operator $f(\lambda) \rightarrow \frac{d}{d \lambda} F(\lambda)$ must be of the form $P=L_{+}^{p / q}$, where the subscript indicates we keep only the differential operator part of a pseudodifferential operator. The nonlinear differential equations $[P, L]=1$ should define nonperturbative $2 D$ quantum gravity coupled to the $(p, q)$ minimal model of conformal field theory. Similarly, the equations for massive models coupled to $2 D$ gravity are of the form $[P, L]=1$, where $P=\sum_{p} t_{p} L_{+}^{p / q}$ and the $t_{p}$ are the "masses" in the theory. Our first task will be to rewrite these equations in first order matrix form.

The $(2 l-1,2)$ Equations: From the work of Drinfeld and Sokolov [18] we can represent the $\mathrm{KdV}$ equations as a Lax pair of first order matrix equations. Let

$$
\mathscr{L} \equiv-\frac{d}{d x}+\left(\begin{array}{cc}
0 & \lambda+u \\
1 & 0
\end{array}\right)
$$

and consider the $\operatorname{sl}(2)$ matrix

$$
\mathscr{P}_{l} \equiv\left(\begin{array}{cc}
A_{l} & B_{l} \\
C_{l} & -A_{l}
\end{array}\right)
$$

where the matrix entries can be expressed in terms of Gelfand-Dickii potentials [19] via

$$
\begin{aligned}
C_{l} & =R_{l}+\lambda R_{l-1}+\cdots+\lambda^{l-1} R_{1}+\lambda^{l} R_{0}, \\
A_{l} & =\frac{1}{2} C_{l}^{\prime}, \\
B_{l} & =(\lambda+u) C_{l}-A_{l}^{\prime} \\
& =4\left(P_{1 l}+\lambda P_{1, l-1}+\cdots+\lambda^{l-1} P_{11}\right. \\
& =2 R_{l+1}-\left(\lambda R_{l}+\lambda^{2} R_{l-1}+\cdots \lambda^{l} R_{1}\right)+\lambda^{l+1} R_{0},
\end{aligned}
$$

where $R_{k} R_{l}^{\prime}=\left(P_{k, l}\right)^{\prime}$ defines $P_{k, l}$ up to a constant. Using the recursion relation one may then verify, $B_{l}^{\prime}+2 A_{l}(\lambda+u)=-2 R_{l+1}^{\prime}$, and hence

$$
\left[\mathscr{P}_{l}, \mathscr{L}\right]=\left(\begin{array}{cc}
0 & -2 R_{l+1}^{\prime} \\
0 & 0
\end{array}\right)
$$


The $l^{\text {th }} \mathrm{KdV}$ flow is thus the compatibility condition $\left[2 \partial / \partial t_{l}+\mathscr{P}_{l}, \mathscr{L}\right]=0$. Similarly, it follows that if we define

$$
\mathbb{P} \equiv-\hbar \frac{d}{d \lambda}+\mathscr{P}_{l}+\left(\begin{array}{cc}
0 & \hbar x+2 R_{l+1} \\
0 & 0
\end{array}\right)
$$

then the equation $\left[\mathbb{P}_{l}, \mathscr{L}\right]=0$ is equivalent to the massless $(2 L-1,2)$ string equation $R_{l+1}=-\frac{1}{2} \hbar x$. In particular

$$
\mathbb{P}_{1}=-\hbar \frac{d}{d \lambda}+\frac{\lambda^{2}}{2}\left(\begin{array}{ll}
0 & 1 \\
0 & 0
\end{array}\right)+\frac{\lambda}{2}\left(\begin{array}{cc}
0 & u / 2 \\
1 & 0
\end{array}\right)+\left(\begin{array}{cc}
-u^{\prime} / 8 & \hbar x+u^{2} / 8 \\
-u / 4 & u^{\prime} / 8
\end{array}\right)
$$

Similarly we can generalize $(2.5)$ to

$$
\mathbb{P}=-\hbar \frac{d}{d \lambda}-\frac{1}{2} \sum_{j}\left(j+\frac{1}{2}\right) T_{j} \mathscr{P}_{j-1}+\left(\begin{array}{cc}
0 & \hbar x-\left(\sum_{j}\left(j+\frac{1}{2}\right) T_{j} R_{j}\right) \\
0 & 0
\end{array}\right)
$$

(where $\mathscr{P}_{-1}=0$ ) and then the compatibility conditions of the linear systems

$$
\begin{aligned}
\mathbb{P} \Psi\left(\lambda, x, T_{j}\right) & =0, \\
\mathscr{L} \Psi\left(\lambda, x, T_{j}\right) & =0, \\
\left(2 \hbar \frac{d}{d T_{j}}+\mathscr{P}_{j}\right) \Psi\left(\lambda, x, T_{j}\right) & =0
\end{aligned}
$$

give the massive $(2 l-1,2)$ and $\mathrm{KdV}$ equations.

The fact that a solution to $\sum\left(j+\frac{1}{2}\right) T_{j} R_{j}=\hbar x$ satisfied the $\mathrm{KdV}$ flow in $T_{j}$ [6] is extremely surprising to those familiar with the almost periodic solutions of $\mathrm{KdV}$, where analogous parameters play the role of moduli of an associated Riemann surface, while the $\mathrm{KdV}$ flows are (straightline) flows along the Jacobian of that surface. We will comment on this relation further below. For now we content ourselves with the following consistency check [6] on (2.7), using the notation of Gelfand-Dickii [19]. Taking derivatives with respect to $x, T_{k}$ and assuming the $\mathrm{KdV}$ flow in $T_{k}$ we have

$$
\begin{aligned}
& \hbar=\sum\left(j+\frac{1}{2}\right) T_{j} R_{j}^{\prime}, \\
& 0=\left(k+\frac{1}{2}\right) R_{k}^{\prime}-\frac{1}{\hbar} \sum_{j}\left(j+\frac{1}{2}\right) T_{j} \xi_{j} R_{k+1}^{\prime},
\end{aligned}
$$

where $\xi_{j}$ are the vector fields generating $\mathrm{KdV}$ flow [19] and we have used commutativity of the flows. The first equation implies $\hbar \delta / \delta u=\sum_{j}\left(j+\frac{1}{2}\right) T_{j} \xi_{j}$, and substitution into the second equation gives $0=\left(k+\frac{1}{2}\right) R_{k}^{\prime}+(\delta / \delta u) R_{k+1}^{\prime}$, a true identity. This verifies consistency. One argument for the $\mathrm{KdV}$ flow was given in [6], we will give another argument below. Note especially that the argument fails for $\hbar=0$.

The $(p, q)$ Equations. In this case we will be somewhat less detailed. Let $L=$ $D^{q}+u_{q-2} D^{q-2}+\cdots$ and consider the generalized $\mathrm{KdV}$ flow $\left[L_{+}^{p / q}, L\right]=d L / d t$. 
We may rewrite this, following Drinfeld-Sokolov, using the operator

$$
\mathscr{L}=D+\Lambda+\left(\begin{array}{ccc}
0 & \ldots & -u_{0} \\
\cdot & \ldots & \cdot \\
\cdot & \ldots & \cdot \\
. & \ldots & \cdot \\
0 & \ldots & -u_{q-2} \\
0 & \ldots & 0
\end{array}\right)
$$

where $\Lambda$ has entries 1 along the lower diagonal and $\lambda$ in the $1, q$ matrix element. Using the methods of [18] one may construct $\mathscr{A}_{q, p}(\lambda)=\Lambda^{p}+\cdots$ such that the generalized $\mathrm{KdV}$ flows are equivalent to

$$
\left.\left.\frac{\partial \mathscr{L}}{\partial t}=[\mathscr{A}) \lambda\right), \mathscr{L}\right]
$$

in particular, there are potentials $R_{q, p ; i}, i=2,3, \ldots q$ generalizing the $R_{l}^{\prime} s$ used above such that

$$
\left[\mathscr{A}_{q, p}, \mathscr{L}\right]=\left(\begin{array}{ccc}
0 & \ldots & -R_{q, p ; q}^{\prime} \\
0 & \ldots & -R_{q, p ; q-1}^{\prime} \\
\cdot & \ldots & \cdot \\
. & \ldots & \cdot \\
0 & \ldots & -R_{q, p ; 2}^{\prime} \\
0 & \ldots & 0
\end{array}\right)
$$

Thus, as before, we may write the string equations as the flatness conditions $\left[\mathbb{B}_{q, p}, \mathscr{L}\right]=0$, where

$$
\left[\mathbb{B}_{q, p}=\hbar \frac{d}{d \lambda}+\mathscr{A}_{q, p}+\left(\begin{array}{ccc}
0 & \ldots & \hbar x-R_{q, p ; q} \\
0 & \ldots & \mathscr{H}_{q-1}-R_{q, p ; q-1} \\
. & \ldots & . \\
. & \ldots & . \\
0 & \ldots & \mathscr{H}_{2}-R_{q, p ; 2} \\
0 & \ldots & 0
\end{array}\right),\right.
$$

and the $\mathscr{H}_{i}$ are constants, analogous to the magnetic field of the Ising model. We may formulate the equations for massive models in the obvious way by taking linear combinations of the $\mathbb{B}_{q, q}$.

Unitary Matrix Models. In [20] equations analogous to the above string equations, but for unitary matrices were derived. It is not obvious whether these equations can be written in Douglas' form $[P, L]=1$. In $[15,21]$ it was shown that they do admit an interpretation as flatness equations. We briefly summarize those equations here for completeness. Periwal and Shevitz showed that the appropriate hierarchy of equations are the self-similar solutions to the MKdV hierarchy. The Lax pair for the MKdV hierarchy may be derived from the above pairs essentially via a Miura transformation. The self-similar solutions are obtained be setting, for the $l^{\text {th }}$ equation, $\psi(x, t)=t^{\alpha} F(z)$, where $z=t^{\alpha} x$ and $\alpha=-1 /(2 l+1)$. Letting $u=f^{2}+f^{\prime}$, the unitary matrix-model hierarchy becomes:

$$
\frac{1}{2} R_{l}[u]^{\prime}-f R_{l}[u]-\frac{z}{2 l+1} f(z)=0 .
$$


Also define $\lambda^{1 / 2}=t^{\alpha \zeta}$. Then after some simple transformations we find the hierarchy (2.9) is the compatibility condition for:

$$
\begin{aligned}
\frac{d}{d z} \Psi+\left(\begin{array}{cc}
\zeta & f \\
f & -\zeta
\end{array}\right) \Psi & =0, \\
\frac{1}{2 l+1} \frac{d}{d \zeta} \Psi+\left[\left(U_{l}+\left(V_{l}^{\prime} / 2 \zeta\right)\right) \sigma_{3}+V_{l} \sigma_{1}+\left(V_{l}^{\prime} / 2 \zeta\right) i \sigma_{2}\right] \Psi & =0,
\end{aligned}
$$

where

$$
\begin{aligned}
U_{l} & \equiv R_{l}+\zeta^{2} R_{l-1}+\cdots \zeta^{2 l} R_{0}+\frac{z}{2 l+1}, \\
V_{l} & \equiv \zeta S_{l-1}+\zeta^{3} S_{l-2}+\cdots \zeta^{2 l-1} S_{0}, \\
S_{l} & \equiv f R_{l}-\frac{1}{2} R_{l}^{\prime},
\end{aligned}
$$

and the GD potentials are evaluated for $u=f^{2}+f^{\prime}$. The particular case of $l=1$ in the above equations gives the PII equation with the normalization $f^{\prime \prime}-2 f^{3}+8 x f / 3=0$. Correlation functions can be discussed in an analogous way to the hermitian matrix model case [21].

General Semisimple Lie Algebras. In [18] Drinfeld and Sokolov wrote analogues of the $\mathrm{KdV}$ equations associated to any Lie algebra $g$. These are again of the form $\dot{\mathscr{L}}=[\mathscr{P}, \mathscr{L}]$, where $\mathscr{L}=\frac{d}{d x}+q+\Lambda, q$ is a function taking values in the Lie algebra and $\Lambda$ is a standard element in the affine Kac-Moody algebra $\hat{g}$. When this algebra is realized as a loop algebra we may again modify $\mathscr{P} \rightarrow \tilde{\mathscr{P}}$ to obtain some equations of the form $0=\left[\frac{d}{d \lambda}+\tilde{\mathscr{P}}, \mathscr{L}\right]$, and these should be the string equations for some matrix model ${ }^{1}$.

Flatness conditions arise very often in physics. The above interpretations suggest, e.g., that possibly one should think about a pure (holomorphic) ChernSimons theory along the lines of [23] with a suitable restriction on the fields. Such an interpretation yields a nice interpretation, e.g., of the $(p, q)$ actions on [24] in terms of Wilson loops. However it is difficult to see why the gauge symmetry should be broken. We will comment again on this below.

\section{String Equations and Isomonodromic Deformation}

The compatibility conditions of the previous section arise naturally in a very interesting problem known as the isomonodromy problem. The theory of isomonodromic deformation has been adequately reviewed in [25-29]. So we confine ourselves here to a very brief description of the method.

Consider a linear homogeneous differential equation

$$
\frac{d \Psi}{d z}=A(z) \Psi
$$

At an irregular singular point $a$ of order $r$ we can write

$$
A(z)=\sum_{n=-r}^{\infty} A_{n}(z-a)^{n-1} \text {. }
$$

\footnotetext{
1 In [5] Douglas suggested they would be associated to the nondiagonal minimal models. This idea
} has been studied in detail in [22] 
Assuming $A_{-r}$ is diagonalizable one can show that there is a formal solution to (3.1) of the form

$$
\Psi \sim\left(\sum_{l \geq 0} \hat{\Psi}^{(l)}(z-a)^{l}\right)(z-a)^{L} e^{T(z-a)}
$$

where $L$ and

$$
T(z-a)=\sum_{i=1}^{r} \frac{T_{r}}{(z-a)^{r}}
$$

are diagonal, and $\hat{\psi}^{(0)}$ in invertible ${ }^{2}$. The analytic meaning of the formal solution (3.2) is that we can divide up a neighborhood of $a$ into sectorial domains $\Omega_{k}=\left\{d_{k}<\arg (z-a)<e_{k}\right\}$ for some constants $d_{k}, e_{k}$ such that in each domain there is a unique true solution $\Psi_{k}$ to (3.1) which is asymptotic to (3.2). On $\Omega_{k+1} \cap \Omega_{k}$ we have $\Psi_{k+1}=\Psi_{k} S_{k}$ for some Stokes matrices. If the differential equation (3.1) depends on parameters we can ask how we may change the parameters so that the "monodromy date" $S_{k}, L$ remains unchanged. As shown in the above references, such questions lead to interesting nonlinear differential equations. We now apply the general formalism of these works to the string equations.

Asymptotic Analysis. Consider the massless $(2 l-1,2)$ equation. In order to perform the asymptotic analysis we follow $[26,30]$ and define $\lambda=\zeta^{2}$ and

$$
\begin{aligned}
& \Psi(\lambda)=\zeta^{1 / 2}\left(\begin{array}{cc}
1 & 1 \\
1 / \zeta & -1 / \zeta
\end{array}\right) W(\zeta), \\
& W(\zeta)=\zeta^{1 / 2} \frac{1}{2}\left(\begin{array}{cc}
1 & \zeta \\
1 & -\zeta
\end{array}\right) \Psi(\lambda),
\end{aligned}
$$

so that $W$ satisfies the differential equation:

$$
\begin{aligned}
\hbar \frac{d W}{d \zeta}= & {\left[\left(B_{l}+\zeta^{2} C_{l}+\Delta_{l}\right) \sigma_{3}-\left(B_{l}-\zeta^{2} C_{l}+\Delta_{l}\right) i \sigma_{2}+\left(2 \zeta A_{l}-\frac{\hbar}{2 \zeta}\right) \sigma_{1}\right] W } \\
= & {\left[\left(\zeta^{2 l+2}+\frac{u^{2}}{8} \zeta^{2 l-2}+\cdots\right) \sigma_{3}+\left(-\frac{u^{\prime}}{4} \zeta^{2 l+1}+\cdots\right) \sigma_{1}\right.} \\
& \left.+\left(-\frac{u}{2} \zeta^{2 l}+\cdots\right)\left(-i \sigma_{2}\right)\right] W
\end{aligned}
$$

where $\Delta_{l}=\hbar x+2 R_{l+1}$. Equation (3.4) has an irregular singularity of order $2 l+3$ at infinity and a regular singularity at the origin. For the massive string equations we replace

$$
\begin{gathered}
C_{l} \rightarrow C \equiv-\frac{1}{2} \sum_{j}\left(j+\frac{1}{2}\right) T_{j} C_{j-1}, \quad \Delta \rightarrow \Delta=\hbar x-\sum\left(j+\frac{1}{2}\right) T_{j} R_{j}, \\
A_{l} \rightarrow \frac{1}{2} C^{\prime}, \quad B_{l} \rightarrow(\lambda+u) C-A^{\prime} .
\end{gathered}
$$

In particular the PI equation is associated with the differential equation:

$$
\hbar \frac{d W}{d \zeta}=\left[\left(\zeta^{4}+\frac{u^{2}}{8}+\hbar x\right) \sigma_{3}-\left(\frac{u}{2} \zeta^{2}+\frac{u^{2}}{8}+\hbar x\right) i \sigma_{2}-\left(\zeta \frac{u^{\prime}}{4}+\frac{\hbar}{2 \zeta}\right) \sigma_{1}\right] W \text {. }
$$

\footnotetext{
2 We state this more carefully in the following section
} 
Returning to the general case, the asymptotic expansion at infinity of a solution $W$ to (3.4) is given by $W \sim \hat{W} e^{T / h}$, where

$$
\begin{aligned}
& T=\left(-\frac{1}{4} \sum_{j=1} T_{j} \zeta^{2 j+1}+\zeta \hbar x\right) \sigma_{3}, \\
& \hat{W}=1+\frac{H_{1}}{\zeta} \sigma_{3}-\frac{u}{4 \zeta^{2}}\left(i \sigma_{2}\right)+\mathcal{O}\left(1 / \zeta^{3}\right)
\end{aligned}
$$

To prove this we observe that we can rewrite $C$ in terms of the resolvent $R(x, \lambda)$ of the Schrödinger operator, $\left(-D^{2}+u+\lambda\right)^{-1}$, used extensively in [19]. In particular,

$$
C=p\left(\zeta ; T_{j}\right) R\left(x, \zeta^{2}\right)+\frac{\hbar x-\Delta}{2 \zeta^{2}}+\mathcal{O}\left(1 / \zeta^{4}\right)
$$

where $p=-\frac{1}{2} \sum\left(j+\frac{1}{2}\right) T_{j} \zeta^{2 j-1}$. Defining $\alpha=\left(2 \zeta^{2}+u\right) R-\frac{1}{2} R^{\prime \prime}, \beta=u R-\frac{1}{2} R^{\prime \prime}$ and $\gamma=\zeta R^{\prime}$ we find that

$$
\hat{W}=\left(\begin{array}{cc}
\zeta+\alpha & -\zeta+\alpha \\
\beta+\gamma & \beta+\gamma
\end{array}\right) D
$$

for any diagonal matrix $D$ diagonalizes equation (3.4) to order $\mathcal{O}(1 / \zeta)$. Equating positive powers of $\zeta$ we find

$$
\frac{\partial T}{\partial \zeta}=(\zeta p+x) \sigma_{3}
$$

from which one obtains the first equation in (3.6). Using the diagonal freedom in defining $\hat{W}$ we can arrange that the expansion in $1 / \zeta$ has the form of the second equation ${ }^{3}$.

Near the origin we may diagonalize the regular singularity to be of the form $-\sigma_{3} /(2 \zeta)$ so that, after a diagonalization the matrix near the origin behaves as $(1+\mathcal{O}(\zeta)) e^{-\frac{1}{2} \log \zeta \sigma_{3}}$. True solutions with given asymptotic behavior will be linked by a connection matrix. Actually, the full extent of the machinery for handling several singular points is not necessary. The original equation in $\lambda$ is regular throughout the $\lambda$ plane. The solution is simply $P \exp \int^{\lambda} A\left(\lambda^{\prime}\right) d \lambda^{\prime}$ for an appropriate matrix $A$, hence the only singularities in $\Psi$ can occur at infinity. Thus, near $\zeta=0$ we have

$$
W(\zeta) \cong \frac{1}{2} \zeta^{-1 / 2}\left(\begin{array}{cc}
1 & \zeta \\
1 & -\zeta
\end{array}\right)
$$

Stokes Matrices. For the $(2 l-1,2)$ string equation we will have $4 l+6$ Stokes sectors $\Omega_{k}$ each containing a unique ray $\theta=\frac{\pi}{4 l+6}(2 k-1), k=0, \ldots 4 l+5$ along which $\cos [(2 l+3) \theta]=0$, thus we may take neighborhoods of infinity defined by:

$$
\Omega_{k} \equiv\left\{\zeta \mid \frac{\pi}{4 l+6}+\frac{\pi}{2 l+3}(k-2)<\arg \zeta<\frac{\pi}{4 l+6}+\frac{\pi}{2 l+3} k\right\}
$$

\footnotetext{
3 Strictly speaking the $\mathcal{O}(1)$ part of the differential equation with this substitution requires $\Delta=0$, which is a condition we will find later for isomonodromic deformation. A more tedious analysis shows that one can start without assuming $\Delta=0$ and derive the same result
} 
for $k=0, \ldots, 4 l+5$. We will consider $k$ as an integer defined modulo $4 l+6$. The Stokes sectors have the property that in $\Omega_{k}$ there is a unique solution $W_{k}$ to (3.4) with the asymptotic behavior $\hat{W} e^{T / \hbar}$. Note that the asymptotic behavior is independent of the sector. On the overlap $\Omega_{k} \cap \Omega_{k+1}$ the two solutions $W_{k+1}$ and $W_{k}$ must be related by

$$
W_{k+1}=W_{k} S_{k}
$$

where the $S_{k}$ are known as Stokes matrices. Since $W_{k+1}$ and $W_{k}$ have the same asymptotic expansion in their respective sectors, the $S_{k}$ are severely constrained. Plugging the asymptotic expansion into $W_{k+1} e^{-T / \hbar}=W_{k} e^{-T / \hbar}\left(e^{T / \hbar} S_{k} e^{-T / \hbar}\right)$ we find $1 \sim e^{T / \hbar} S_{k} e^{-T / \hbar}$, and taking account of the sign of the real part of the leading term in the essential singularity we find:

$$
S_{2 k}=\left(\begin{array}{cc}
1 & 0 \\
S_{2 k} & 1
\end{array}\right), \quad S_{2 k+1}=\left(\begin{array}{cc}
1 & s_{2 k+1} \\
0 & 1
\end{array}\right)
$$

In fact, from the symmetry of Eq. (3.4) we may conclude that $W_{k+2 l+3}(\zeta)=$ $\sigma_{1} W_{k}(-\zeta) \sigma_{1}$, and hence that $S_{k+2 l+3}=\sigma_{1} S_{k} \sigma_{1}=S_{k}^{\text {tr }}$, i.e., $s_{k+2 l+3}=s_{k}$. By (3.9) $W$ has monodromy -1 upon analytic continuation around zero (or infinity), implying a constraint on the Stokes matrices. In fact, a stronger constraint may be derived from the fact that, again by (3.9), if we analytically continue $W_{1}$ from $\Omega_{1}$, then $W_{1}(-\zeta)=-i \sigma_{1} W_{1}(\zeta)$, hence

$$
S_{1} \ldots S_{2 l+3}=-i \sigma_{1} .
$$

Hence, the Stokes parameters are not alle independent. Since the determinant of (3.13) is automatically satisfied, (3.13) only imposes three independent constraints on the Stokes matrices, so we have $2 l+3-3=2 l$ independent Stokes parameters. Finally, we may consider the constraint on the Stokes parameters imposed by reality of $u$. If $u$ is real then all the coefficients of powers of $\zeta$ in $\mathbb{P}$ are real so that $\bar{W}_{k}(\zeta)=W_{-k}(\bar{\zeta})$. From this it follows that for real solutions $s_{2 l+3-k}=-\bar{s}_{k}$.

Isomonodromic Deformation. We now interpret the compatibility conditions of the previous section as the conditions required for isomonodromy under changes of $x, T_{j}$. In the literature [25-27, 29] on isomonodromic deformation the Stokes parameters $s_{j}$ are referred to as monodromy parameters ${ }^{4}$. The problem of isomonodromic deformation is the problem of finding conditions on the $u, u_{x}, u_{x x}, \ldots$ (considered as independent numbers) such that if we change $x, T_{j}$ the Stokes matrices remain fixed. We will give both a geometric and a physical description of the isomonodromy problem in subsequent sections. For the moment let us simply consider the solution. Under a deformation of $x, T_{j}$ the solution $W$ of (3.4) will become a smooth function of the $x, T_{j}$. Since $\Psi$ is regular throughout the $\lambda$ plane we know that the differential $d \Psi \Psi^{-1}$ is a matrix of holomorphic one-forms whose only (rational) singularity lies at infinity, and is thus uniquely determined by that singularity. Since the Stokes data are assumed to be $x$-indepedent we can

\footnotetext{
4 One also considers the formal monodromy, i.e., the matrix $T_{\infty}$ multiplying the term with $\log \zeta$ in $T$ as a monodromy parameter. In our problem we consider this parameter to be set equal to $1 / 2$ always
} 
substitute the asymptotic expansion and compute

$$
\begin{aligned}
\frac{\partial \Psi}{\partial x} \Psi^{-1} & =\left(\begin{array}{cc}
1 & 1 \\
1 / \zeta & -1 / \zeta
\end{array}\right)\left[\left(\zeta+H_{1}^{\prime} / \zeta\right) \sigma_{3}+\frac{u}{2 \zeta}\left(\begin{array}{cc}
0 & -1 \\
1 & 0
\end{array}\right)\right] \\
& \times \frac{1}{2}\left(\begin{array}{cc}
1 & \zeta \\
1 & -\zeta
\end{array}\right) \bmod (1 / \zeta) \\
& =\left(\begin{array}{cc}
0 & \lambda+H_{1}^{\prime}+(u / 2) \\
1 & 0
\end{array}\right) .
\end{aligned}
$$

Consistency with the $\lambda$ equation then forces $H_{1}^{\prime}=u / 2$. In terms of $W$ we have:

$$
\frac{\partial W}{\partial x} W^{-1}=\zeta \sigma_{3}+\frac{u}{2 \zeta}\left(\begin{array}{ll}
1 & -1 \\
1 & -1
\end{array}\right) \text {. }
$$

(If we had worked directly with $W$ then in computing $W_{x} W^{-1}$ we would have had to drop only terms of order $\mathcal{O}\left(1 / \zeta^{2}\right)$.)

Similarly, requiring that the Stokes parameters and the formal monodromy be independent of the $T_{j}$ leads to the equations:

$$
\frac{\partial W}{\partial T_{j}} W^{-1}=\left[\frac{1}{\zeta} \frac{\partial H}{\partial T_{j}} \sigma_{3}-\frac{1}{4} \zeta^{2 j+1} \hat{W} \sigma_{3} \hat{W}^{-1}\right] \bmod 1 / \zeta^{2}
$$

Using (3.8), which holds to $\mathcal{O}\left(\zeta^{-(2 l+1)}\right)$ we establish the third equation in (2.7). The compatibility of the $x, T_{j}$ flows now shows that $u$ satisfies the $\mathrm{KdV}$ hierarchy.

$r$ Functions. In $[25,26]$ it was shown that if we have an isomonodromic deformation problem then one can naturally define a closed one-form on the space of deformation parameters:

$$
\omega=\operatorname{Res}_{\zeta=\infty} \operatorname{tr}\left[\left(\hat{W}^{-1} \frac{d \hat{W}}{D \zeta}\right) d T\right] .
$$

If there is more than one singular point then we must sum over the residues, using the appropriate asymptotic expansion near each singular point. Since $\omega$ is closed one can then define (locally, in the space of deformation parameters) the tau function via $\omega=d(\log \tau)$.

We will now show that $u\left(x ; T_{j}\right)$ (and hence, the free energy of the matrix model) is the second logarithmic derivative of the tau function, just as in the case of quasiperiodic and soliton solutions to the $\mathrm{KdV}$ equations. We have just seen that $d H_{1} / d x=u$, and substituting into the equation for $d(\log \tau)$ we get $d(\log \tau)=-2 H_{1}$, hence

$$
u\left(x ; T_{j}\right)=-\frac{d^{2}}{d x^{2}} \log \tau
$$

Since the tau function for isomonodromic deformations is known always to be holomorphic [31] we see that the only singularities of $u$ are second order poles. (As one may easily check directly from the differential equation, the coefficient of a second order pole must be 2 , implying that our $\tau$ function must be the square of a holomorphic function. This is naturally explained by the occurrence of a pair of Weyl fermions in the free fermion formulation below.)

Inverse Monodromy Problem and Initial Conditions. As we have seen, under isomonodromic deformation the monodromy data are in fact indepedent of the 
parameters $x, T_{j}$. On the other hand, in order to construct the differential equation and solve explicitly for the monodromy data we must begin with an actual solution $u\left(x ; T_{j}\right)$ of the string equations. It turns out that we may identify the $2 l$ initial conditions needed to define a solution of the string equations as the data needed to fix completely the differential equation. Strictly speaking then, we should speak of the function $u\left(x ; T_{j}, s_{j}\right)$ since all these data are needed to determine $u$.

The problem of determining explicitly the data $s_{i}$ in terms of a solution (or in terms of its asymptotics) is known as the direct monodromy problem, while the problem of finding the inverse relation, i.e., given the $s_{i}$ find $u(x)$ (in particular, its initial conditions) is known as the inverse monodromy problem. These issues have been well-studied in the lierature [29]. It is not always true that the inverse monodromy problem is solvable. For instance, an unfortunate choice of initial conditions in the form of the $s_{i}$ and the parameter $x$ could put us on top of a pole for that function $u(x)$ determined by the $s_{i}{ }^{5}$. The existence of this pole, whose physical implications have been widely discussed $[1,6,32,33]$ can be given a geometrical interpretation in terms of the nontriviality of a certain vector bundle in the case of the PII equation [34]. We expect a similar statement will hold for the family of equations discussed here.

The $(p, q)$ Equations. The above discussion may be generalized to the entire set of $(p, q)$ string equations. Again we must diagonalize the leading singularity. To do this, choose a $q^{\text {th }}$ root of $\lambda$, call it $\xi$ so that the other roots are $\xi_{k}=\omega^{k-1} \xi$, where $\omega=e^{2 \pi i / q}, k=1, \ldots q$. Then we take

$$
\Xi \equiv\left(\begin{array}{ccc}
\xi_{1}^{q-1} & \ldots & \xi_{q}^{q-1} \\
\xi_{1}^{q-2} & \ldots & \xi_{q}^{q-2} \\
\cdot & \ldots & \cdot \\
\xi_{1} & \ldots & \xi_{q} \\
1 & \ldots & 1
\end{array}\right)
$$

so that $\Xi^{-1} \Delta \Xi=\Omega \equiv \operatorname{Diag}\left\{1, \omega, \ldots, \omega^{q-1}\right.$. Therefore, defining $W(\xi)=\Xi^{-1} \Psi$ we obtain the equation

$$
-\frac{d W}{d \xi}=\left(q \xi^{p+q-1} \Omega^{p}+\mathcal{O}\left(\xi^{p+q-3}\right)\right) W .
$$

From the asymptotic expansion we find again that $W=\hat{W} e^{T / \hbar}$ with

$$
\begin{aligned}
T & =\frac{-q}{p+q} \Omega^{p} \xi^{p+q}-\hbar \xi x \Omega, \\
\hat{W} & =1+\frac{H_{1}}{\xi} \Omega^{-1}+\frac{W_{2}}{\xi^{2}}+\cdots,
\end{aligned}
$$

and for isomonodromic deformation we have $u_{q-2}=q H_{1}^{\prime}$. It then follows that

$$
\begin{aligned}
\frac{d}{d x} \log \tau & =\operatorname{Res}_{\xi=\infty} \operatorname{tr}\left[\hat{W}^{-1} \frac{\partial \hat{W}}{\partial \xi}\left(\frac{\partial T}{\partial x}\right)\right] \\
& =q H_{1},
\end{aligned}
$$

\footnotetext{
5 The fact that such a pole is the only possible obstruction will be obvious, if not rigorous, from the free fermion point of view below
} 
so that

$$
u_{q-2}=\frac{\partial^{2}}{\partial x^{2}} \log \tau
$$

is always true. Hence the only singularities for a solution to any of the string equations are second order poles.

A similar analysis can be carried out for the unitary matrix models [21]. In this case one finds that if $f$ is determined by (2.9) then $f^{2}=\frac{\partial^{2}}{\partial x^{2}} \log \tau$. Since $f^{2}$ is the specific heat of the matrix model we see once again that the partition function is a tau function.

\section{The Moduli Space of the String Equations}

In this section we will give our first geometrical interpretation of the physical parameters determining the connection $\mathbb{P}$, that is, the parameters $T_{j}$ and the initial conditions of the equation for $u(x)$. They will be seen to be moduli for a certain class of meromorphic gauge potentials.

Consider a meromorphic gauge potential $A_{z}(z)$ on the complex plane. $A_{z}$ defines a connection on a (trivial) bundle over $\mathbb{C}$. Consider the problem of classifying $A$ up to gauge equivalence. The resulting classification depends crucially on the nature of the singularities of $A$ and on the admissible class of gauge transformations. For example, in the local theory we take the possible (isolated) singularity of $A$ to be at the origin. If $A$ has a regular singular point, i.e., if $A$ has a simple pole then, up to equivalence under meromorphic gauge transformations, $A$ is classified by the conjugacy class of the monodromy $P \exp \int A$ around zero. This follows since if we try to gauge $A$ to zero by solving the equation $d \Psi / d z=A \Psi$ then there is a solution of the form $\Psi=\hat{\Psi}(z) z^{M}$, where $\hat{\Psi}$ is holomorphic near zero, and $e^{2 \pi i M}$ is the monodromy matrix. Passing to the global theory of a connection on a vector bundle over a Riemann surface $\Sigma$, the solution to the famous Riemann-Hilbert problem states that the moduli space of connections with regular singularities at $a_{i}$ are classified by the conjugacy classes $\operatorname{Hom}\left(\pi_{1}\left(\Sigma-\left\{a_{i}\right\}\right), G L(m, \mathbb{C})\right) / \sim$. Related facts have been used extensively in investigations of Chern-Simons-Witten theory.

The situations is very different, and far more complicated, for connections with irregular singularities, i.e., with higher order poles. We briefly summarize the situation, see [35-42] for the full story. We follow the presentation of $[40,42]$. A major result in the theory of differential equations, the Hukuhara-Turittin formal reduction theorem, states that if $A$ has an irregular singular point at $z=0$, we may solve $d \Psi / d z=A \Psi$ with $\Psi=\hat{\Psi} e^{\Lambda}$, where $\hat{\Psi}$ is a formal asymptotic series and

$$
\frac{d \Delta}{d z}=D_{r_{1}} z^{r_{1}}+D_{r_{2}} z^{r_{2}}+\cdots D_{r_{m}} z^{r_{m}}+z^{-1} C
$$

where $r_{i}$ are rational numbers with $r_{1}<\cdots r_{m}<-1, D_{r_{1}}$ are diagonal constant matrices and $C$ commutes with the $D_{r_{i}}$. (See. e.g., [35], Theorem 19.1 for a careful statement and proof of this.) Thus, a gauge transformation by $\hat{\Psi}$ transforms the connection to the above canonical form. Moreover, the numbers $r_{i}$ and the conjugacy class of $\left\{D_{r_{i}}, \exp [2 \pi i k C]\right\}$ are gauge invariant. These are therefore the moduli of meromorphic gauge fields under thr orbits of formal gauge transformations. Applying the above observations to the class of gauge potentials defined 
by (3.4) and its generalizations we see that the $\left\{T_{j}, x\right\}$ should be thought of as such moduli.

If we now ask for the orbits under meromorphic gauge transformations, i.e., transformations with a convergent Laurent expansion near zero then, as shown in [36-40], one must take into account a more subtle invariant. This is the collection of Stokes matrices, or more properly, a Stokes cocycle, which we now describe $^{6}$. Suppose $A$ is in the formal equivalence class (4.1) and $A$ gives rise to Stokes matrices $S_{k}$ in sectors around $z=0$. We can then consider the matrices $\mathscr{S}_{k}(z)=\exp (\Delta(z)) S_{k} \exp (-\Lambda(z))$, defined in each sector $\Omega_{k}$. These satisfy

$$
\begin{aligned}
\mathscr{S}_{k} & \sim 1 \text { in } \Omega_{k}, \\
\frac{d \mathscr{S}_{k}}{d z} & =\left[\frac{d \Lambda}{d z}, \mathscr{S}_{k}\right] .
\end{aligned}
$$

Let us now identify a sector $\Omega=\left\{\theta_{1}<\arg z<\theta_{2}\right\}$ with the corresponding region on the unit circle. In this way we can define a sheaf ${ }^{7}, \operatorname{st}(\Lambda)$, over $S^{1}$ whose sections consist of matrices satisfying the two conditions in (4.2). The main theorem of Sibuya-Malgrange states that the nonabelian sheaf cohomology group $H^{1}\left(S^{1}, s t(\Lambda)\right)$ is isomorphic to the moduli space under meromorphic gauge equivalence of connections formally equivalent to (4.1).

In the examples studied in this paper we may represent the Stokes cocycle by the matrices:

$$
\left(\begin{array}{cc}
1 & e^{2 T / \hbar} s_{2 k+1} \\
0 & 1
\end{array}\right), \quad\left(\begin{array}{cc}
1 & 0 \\
e^{-2 T / \hbar} s_{s k} & 1
\end{array}\right)
$$

in $\Omega_{2 k+2} \cap \Omega_{2 k+1}$ and $\Omega_{2 k+1} \cap \Omega_{2 k}$ respectively. The moduli in this case are simply the Stokes parameters $s_{i}$ and hence the initial conditions for the solution $u(x)$ to the string equations. Hence the physical data - the masses $T_{j}$ and the initial conditions, as expressed through the Stokes parameters $s_{j}$ are coordinates on the moduli space of a class of meromorphic gauge fields under meromorphic gauge equivalence. We think that it is an interesting question to elucidate what distinguishes potentials associated with integrable systems in the space of all connections with irregular singular points. This is the same as the problem mentioned at the end of Sect. 2.

\section{Quantum Riemann Surfaces}

In this section we will examine more closely the geometry of the vector bundles defined in Sect.3. In particular we will be interested in the following question. It is well known that a pair of commutating differential operators $[P, L]=$ 0 leads, via the Burchnall-Chaundy-Krichever theory, to the construction of an algebraic curve $\Sigma$ with a line bundle $^{8} \mathscr{L} \rightarrow \Sigma$. The $\mathrm{KdV}$ flows are then just straightline motion of $\mathscr{L}$ along the Picard variety of $\Sigma$. Since the string equations can be formulated as $[P, L]=\hbar$, one is naturally lead to ask if there is a sense in which one may associate to solutions of these equations a

\footnotetext{
6 We skip over many technical details in what follows. The interested reader should consult the above references

7 of unipotent groups

8 If $\Sigma$ in singular $\mathscr{L}$ is not a line bundle because of identifications at the singular points, instead it is a "coherent sheaf." We will assume below that our Riemann surfaces are nonsingular
} 
corresponding "quantum" geometry. We will propose such a notion below, based on isomonodromic deformation".

Although the BCK theory has already been beautifully described in $[13,43$, 44] and elsewhere, we review it again from a slightly different point of view better suited to our purposes.

We work with the $\mathrm{KdV}$ hierarchy for simplicitly, and will indicate the proper generalizations to the generalized $\mathrm{KdV}$ hierarchies later. The usual presentation of the BCK theory begins with the band theory of the Schrödinger operator $L=D^{2}-u(x)$. We consider the two-dimensional eigenspace of $L$ :

$$
V_{\lambda}=\{\psi(x) \mid L \psi(x)=\lambda \psi(x)\} .
$$

Eigenfunctions can be shown to be meromorphic functions of the "momentum" $z$ defined by $\lambda=z^{2}$. More precisely, one introduces the Baker-Akhiezer (BA) eigenfunction which is uniquely characterized by a normalization condition $\psi\left(x_{0}, z\right)=1$, for some fixed $x_{0}$, and the expansion near $z=\infty$,

$$
\psi(x, z)=e^{\left(x-x_{0}\right) z}\left[1+\frac{a_{1}(x)}{z}+\frac{a_{2}(x)}{z^{2}}+\cdots\right] .
$$

The BA function has a meromorphic extension to an affine hyperelliptic curve $\Sigma_{0}$ double covering the affine $\lambda$ line $\mathbb{C}$, and is a section of a (trivialized) line bundle $\mathscr{L} \rightarrow \Sigma_{0}$. For our purposes it is more convenient to work with vector bundles on $\mathbb{C}$ rather than line bundles on $\Sigma_{0}$. The $V_{\lambda}$ define a trivial bundle over the affine $\lambda$-line. We will be concerned with various framings of this bundle, which are in one-one correspondence with the invertible solutions of

$$
\mathscr{L} \Psi=\left[-\frac{d}{d x}+\left(\begin{array}{cc}
0 & \lambda+u \\
1 & 0
\end{array}\right)\right] \Psi=0,
$$

since a solution is given by the Wronskian matrix

$$
\Psi(x)=\left(\begin{array}{ll}
\psi_{1}^{\prime} & \psi_{2}^{\prime} \\
\psi_{1} & \psi_{2}
\end{array}\right)
$$

where $\psi_{1}, \psi_{2}$ are two linearly independent elements of $V_{\lambda}$. A standard frame for this bundle is provided by the two solutions $\phi_{i}\left(x ; x_{0}, \lambda\right), i=1,2$, which satisfy $\phi_{i}^{(j-1)}\left(x_{0} ; x_{0}, \lambda\right)=\delta_{i j}$. The $\phi_{i}$ are entire functions of $\lambda$.

Recall that if $u(x)$ solves the stationary $\mathrm{KdV}$ equations:

$$
[P, L]=0, \quad P=\sum c_{l} L_{+}^{(2 l+1) / 2},
$$

then $P$ restricts to $V_{\lambda}$ and its restriction in the $\phi_{i}$ basis is a matrix which is polynomial in $\lambda$ and a differential polynomial in $u\left(x_{0}\right)$. In fact, letting $P_{l}=L_{+}^{(2 l+1) / 2}$ one may easily show that

$$
\mathscr{P}_{l}[u(x)] \Psi=\left(\begin{array}{cc}
\left(P_{l} \psi_{1}\right)^{\prime} & \left(P_{l} \psi_{2}\right)^{\prime} \\
P_{l} \psi_{1} & P_{l} \psi_{2}
\end{array}\right)
$$

\footnotetext{
9 This is not to be confused with the fact that the stationary $\mathrm{KdV}$ equations themselves can be written as an isomonodromic deformation problem. We discuss this latter formulation of stationary $\mathrm{KdV}$ briefly in Sect. 6
} 


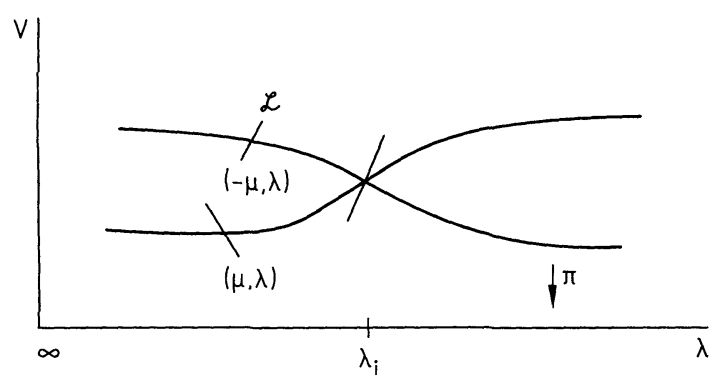

Fig. 1. A schematic drawing of the Riemann surface and the Krichever line bundle defined by the BA framing, in the neighborhood of a branch point

from which it follows that

$$
P_{l}:\left(\begin{array}{c}
\phi_{1} \\
\phi_{2}
\end{array}\right) \rightarrow\left(\begin{array}{cc}
-A_{l}\left[u\left(x_{0}\right)\right] & B_{l}\left[u\left(x_{0}\right)\right] \\
C_{l}\left[u\left(x_{0}\right)\right] & A_{l}\left[u\left(x_{0}\right)\right]
\end{array}\right)\left(\begin{array}{c}
\phi_{1} \\
\phi_{2}
\end{array}\right) .
$$

The eigenvalues of $\left.P_{l}\right|_{V_{\lambda}}$ thus satisfy the characteristic equation of this matrix, which defines an affine curve $\Sigma_{0}$ by the equation $\mu^{2}=A^{2}+B C$. (Recall that $A, B, C$ are polynomials in $\lambda$ and differential polynomials in $x_{0}$. It is a simple consequence of (5.4) that the determinant $A^{2}+B C$ is independent of $x_{0}$.) Consider a patch $U$ in $\mathbb{C}$. If $U$ is simply connected and does not contain any of the roots of $\mu(\lambda)$, then we may choose a framing which diagonalizes $P_{l}$ over $U$. The eigenspaces associated to $\pm \mu$ canonically define lines in the pullback bundle $\pi^{*} V$, where $\pi:(\mu, \lambda) \rightarrow \lambda$ is the projection. We may choose, e.g., $\phi_{ \pm \mu, \lambda}=C \phi_{1}+( \pm \mu+A) \phi_{2}$. (One can impose the normalization condition $\phi_{\mu, \lambda}\left(x_{0}\right)=1$. In this case the framing will be meromorphic.) At a branch point $\lambda_{i}$, where $\mu\left(\lambda_{i}\right)=0$ the two eigenspaces degenerate to a single line, so that we can say the $\mu$ eigenspace of $P_{l}$ at the point $(\mu, \lambda)$, defines a holomorphic line bundle $\mathscr{L} \rightarrow \Sigma_{0}$. The situation is summarized in Fig. $1^{10}$. Finally, note that for large $\lambda$ the eigenfunctions must be plane waves, $\sim e^{ \pm x z}$, and since $P$ is a differential operator of odd order we must have asymptotics $\phi_{\mu, \lambda}(x) \sim e^{ \pm x z}$, with the choice of sign depending on $\mu$, so that these eigenfunctions are proportional to the BA functions $\psi(x, \pm z)$.

Let us define a Baker-Akhiezer framing $\Psi$ to be a simultaneous solution of (5.2) and

$$
\mathscr{P} \Psi=\Psi \sigma_{3} \mu(\lambda)
$$

which has the asymptotics

$$
\Psi \sim \hat{\Psi} e^{\left(x-x_{0}\right) z \sigma_{3}}
$$

where $\hat{\Psi}$ has a power series expansion in $1 / z$ near $\lambda \rightarrow \infty$, and is nonsingular except at the branch points $\lambda_{i}$ and the zeroes of $C$. For example, choosing $\psi_{1,2}=\psi(x, \pm z)$ in (5.3) gives a normalized BA framing. A BA framing is singlevalued and meromorphic on $\Sigma_{0}$ but not on the $\lambda$ line. Rather, upon analytic continuation around a branch point we have the monodromy

$$
\Psi \rightarrow \Psi \sigma_{1}
$$

\footnotetext{
10 The situation can also be summarized in the sheaf-theoretic formula: $0 \rightarrow \pi * \mathscr{L} \rightarrow V \rightarrow \oplus P S_{P} \rightarrow 0$, where $\pi * \mathscr{L}$ is the direct image and $S_{P}$ are sheaves supported on branch points
} 
reflecting the interchange of the two sheets. Nevertheless, we can construct our Riemann surface $\Sigma_{0}$ working completely with the framing for a bundle $V \rightarrow \mathbb{C}$ and $\Sigma_{0}$ can be pictured as "sitting inside the total space of $V$," in the sense that if we consider the surface in $\mathbb{C} \times V$ swept out by the tips of the frame we obtain a copy of $\Sigma_{0}$ as in Fig. 1 . Since the date defining a BA framing is essentially the data defining the famous quintuplets occurring in Krichever's construction [13] we expect that one can define a "moduli space of BA framings," which will be, essentially, the universal Jacobian over the moduli space of curves.

We have phrased the BCK theory in such a way that we can now describe its noncommutative, or quantum, analog associated to the equation $[P, L]=\hbar$. The main claim is that the analog of the BA framing is a solution $\Psi(x, \lambda)$ to

$$
\mathscr{L} \Psi=0, \quad \mathbb{P}_{l} \Psi=0,
$$

and that in the $\hbar \rightarrow 0$ limit this framing reproduces the $\mathrm{BA}$ framing in the following sense.

The gauge connection $\left(\mathscr{L}, \mathbb{P}_{l}\right)$ is flat, so an entire solution of $(5.10)$ exists for finite values of $x, \lambda$. Morally speaking, $\Psi$ defines a global framing of " $\pi * V$ ", where $\pi$ should be a projection from a "quantum Riemann surface." We can try to get a picture of this object by studying the framing over the $\lambda$ line, in the semiclassical $\hbar \rightarrow 0$ limit. To this end let us consider a family of solutions $u_{\hbar}(x)$ to the string equations $\sum\left(j+\frac{1}{2}\right) T_{j} R_{j}=\hbar x$ which have a smooth $\hbar \rightarrow 0$ limit. For example, in the case of PI the asymptotic analysis of Boutroux shows that $u_{h}$ approaches either a constant or the Weierstrass $\wp$ function. In general we can write $u=u^{(0)}+\hbar u^{(1)}+\cdots$. It will also be of interest to let the equation itself vary with $\hbar$, so we also let $T_{i}=T_{i}^{(0)}+\hbar T_{i}^{(1)}+\cdots$. (Effectively, we are working over the ring $\mathbb{C}[[\hbar]]$.) For such a family we can study the equation $\mathbb{P} \Psi=0$, i.e.,

$$
\hbar \frac{\partial \Psi}{\partial \lambda}=\left(-\frac{1}{2} \sum_{j}\left(j+\frac{1}{2}\right) T_{j} \mathscr{P}_{j-1}[u]\right) \Psi
$$

via the WKB approximation using an analysis similar to that of Appendix A. We first summarize the main conclusion of the analysis: In the $\hbar \rightarrow 0$ limit the WKB turning points become the branch points $\lambda_{i}$ of the curve $\Sigma_{0}$ and a choice of Stokes ray emanating from each of these points becomes a branch cut for the affine hyperelliptic curve $\Sigma_{0}$ defined by $\left\{\left(\mu_{0}, \lambda\right)\right\}$ where $\pm \mu_{0}$ are the eigenvalues of $\sum\left(j+\frac{1}{2}\right) T_{j} P_{j-1}\left[u^{(0)}\right]$ acting on $V_{\lambda}$. In the various WKB sectors, and outside a neighborhood (vanishing with $\hbar$ ) of the branch cuts we may find solutions $\Psi_{i}$ of $(5.10)$ so that if we define:

$$
\Psi_{i}(\lambda) \equiv \tilde{\Psi}_{\hbar}^{(i)}(\lambda) \exp \left[\frac{\sigma_{r}}{\hbar} \int_{\lambda_{i}}^{\lambda} \mu_{0}\left(\lambda^{\prime}\right) d \lambda^{\prime}\right],
$$

then $\tilde{\Psi}_{\hbar}^{(i)}$ has a smooth classical limit, and is, in fact, a BA framing in the appropriate sector. Note that it immediately follows from the smoothness of $\tilde{\Psi}$ that if we substitute (5.12) into (5.11) then in the limit $\tilde{\Psi}_{\hbar} \rightarrow \tilde{\Psi}$ the differential equation (5.11) becomes the eigenvalue condition (5.7):

$$
-\frac{1}{2}\left(\sum\left(j+\frac{1}{2}\right) T_{j} \mathscr{P}_{j-1}\left[u^{(0)}\right]\right) \tilde{\Psi}=\tilde{\Psi} \sigma_{3} \mu_{0}(\lambda) .
$$




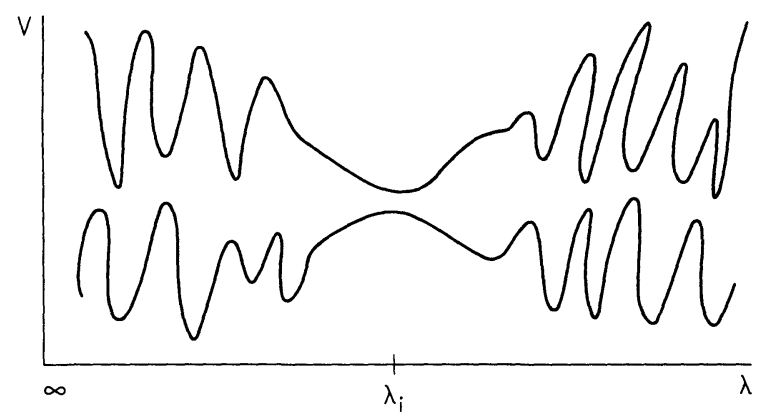

Fig. 2. A schematic drawing of a framing defined by the equation in $\hbar$ in the neighborhood of a branch point. The oscillations are along the directions in $V_{\lambda}$ defined by the framing in Fig. 1

One surprise we find is that the BA framings defined in the different WKB sectors differ by multiplication on the right by nontrivial (i.e., nondiagonal) matrices, a reflection of the Stokes phenomenon. Thus, heuristically speaking, we can picture a quantum Riemann surface as a widely fluctuating trivialization of a vector bundle which becomes smooth, and looks like a classical Riemann surface, in the neighborhood of the branch points as in Fig. 2. The fluctuations are always directed along the line of the Krichever line bundle. Because of the Stokes phenomenon, even when we factor out the wild oscillations along the Krichever line bundle, we find that in different sectors of $\mathbb{C} \times V$ the Riemann surface as been "rotated" and "sheared." 11 We now provide some justification of this picture.

WKB Analysis. We write (5.11) as

$$
\hbar \frac{\partial \Psi}{\partial \lambda}=\left(\alpha \sigma_{3}+\beta \sigma_{2}+\gamma \sigma_{1}\right) \Psi \equiv \mathscr{A} \Psi
$$

where $\alpha=A, \gamma+i \beta=C, \gamma-i \beta=B$. Note that $\mathscr{A}$ is $\mathcal{O}(1)$ as $\hbar \rightarrow 0$. The eigenvalues of $\mathscr{A}$ are given by $\mu^{2}=\alpha^{2}+\beta^{2}+\gamma^{2}=A^{2}+B C\left[u_{h}\right]$ and approach $\mu_{0}^{2}$ smoothly in the classical limit. Therefore, writing $\mu_{0}^{2}=\Pi\left(\lambda-\lambda_{i}\right)$ we must define $3(2 l+1)$ conjugate Stokes lines by the vanishing real part:

$$
\Re \int_{\lambda_{l}}^{\lambda} \mu_{0}\left(\lambda^{\prime}\right) d \lambda^{\prime}=0 .
$$

These are similar to the Stokes lines in Appendix A. In this case the integrand vanishes as $\mathcal{O}\left(\lambda-\lambda_{i}\right)^{1 / 2}$ at the points $\lambda_{i}$. Hence there are three lines emerging from each of these points at angles $2 \pi / 3$. We can get a picture of the Stokes lines, at least, for some region of moduli space, by first considering the case in which $\mu^{2}=\prod_{i=1}^{2 l+2}\left(\lambda-\lambda_{i}\right)$ with all the $\lambda_{i}$ real, with $\lambda_{i}<\lambda_{i+1}$. Then one can easily show that the Stokes lines have the form given in Fig. 3. The proof proceeds as follows. We know that as $\lambda \rightarrow \infty$ we must reproduce the full set of $2 l+3$ Stokes rays going to infinity. Moreover, whenever two branch points coalesce the triple

\footnotetext{
11 The resulting object is thus somewhat reminiscent of certain cubist paintings of Picasso and Braque
} 


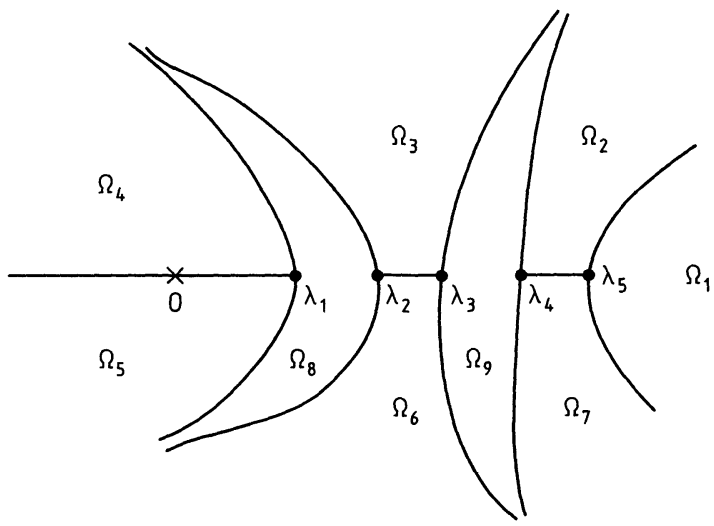

Fig. 3. An example of conjugate Stokes lines in the case where the branch points are all real. In this case the surface has genus two

joining points for Stokes lines must turn into quadrupule joining points. Finally, the intervals on the real axis are certainly among the Stokes lines. The above picture is uniquely determined by these criteria. We may now imagine moving the branch points off the real axis. The picture will deform smoothly unless two branch points collide or unless a branch point hits a Stokes line. In that case we may expect fairly intricate phenomena which is outside the scope of this paper. In general the lines will divide the plane into several regions $\Omega_{k}$.

Our first goal is to establish the following result. In the interior of each region $\Omega_{k}$, for $\left|\lambda-\lambda_{i}\right| \geq \mathcal{O}\left(\hbar^{2 / 3}\right)$ there are solutions $\Psi_{i}(x, \lambda)$ to $(5.10)$ such that

$$
\lim _{\hbar \rightarrow 0} \Psi_{i}(x, \lambda) \exp \left[-\frac{\sigma_{3}}{\hbar} \int_{\lambda_{i}}^{\lambda} \mu_{0}\left(\lambda^{\prime}\right) d \lambda^{\prime}\right]
$$

exists and is a BA framing.

To prove this, recall that the usual theorem in asymptotic analysis guarantees that there will be a solution $\Psi_{i}$ in $\Omega_{i}$ with the asymptotic expansion

$$
\Psi_{i} \sim L_{i}\left(1+\hbar N_{i}^{(1)}+\cdots\right) \exp \left[\frac{\sigma_{3}}{\hbar} \int_{\lambda_{i}}^{\lambda} \mu_{\hbar}\left(\lambda^{\prime}\right) d \lambda^{\prime}\right]
$$

where

$$
\mathscr{A} L_{i}=L_{i} \sigma_{3} \mu_{h},
$$

and, if we expand $L_{i}=L_{i}^{(0)}+\hbar L_{i}^{(1)}+\cdots$, then we require:

$$
\operatorname{Diag}\left\{\left(L_{i}^{(0)}\right)^{-1} \frac{\partial L_{i}^{(0)}}{\partial \lambda}\right\}=0 \text {. }
$$

Therefore, we study the difference of the WKB phase factors:

$$
F_{i}\left(\lambda, x ; T_{j}\right) \equiv \lim _{\hbar \rightarrow 0}\left[\frac{1}{\hbar} \int_{\lambda_{i}}^{\lambda} \mu_{\hbar}\left(\lambda^{\prime}\right) d \lambda^{\prime}-\frac{1}{\hbar} \int_{\lambda_{i}}^{\lambda} \mu_{0}\left(\lambda^{\prime}\right) d \lambda^{\prime}\right]
$$


If $\mu^{2}$ has a simple zero then it is easy to see the limit exists.

Since the most general BA framing differs from the normalized BA framing by right-multiplication by a diagonal matrix depending only on $\lambda$ and having power series asymptotics at infinity, we can establish the result by finding a suitable function $f_{i}(\lambda)$ such that

$$
L_{i}^{(0)}=\left(\begin{array}{ll}
\phi_{\mu, \lambda}^{\prime} & \phi_{-\mu, \lambda}^{\prime} \\
\phi_{\mu, \lambda} & \phi_{-\mu, \lambda}
\end{array}\right)\left(\begin{array}{ll}
f_{i} e^{-F_{i}} & \\
& f_{i}^{-1} e^{F_{i}}
\end{array}\right),
$$

where we normalize the first matrix to have unit Wronskian. We determine $f_{i}$ by

$$
\frac{\partial}{\partial \lambda} \log f_{i}=\frac{\partial F_{i}}{\partial \lambda}-\left(\phi_{-\mu, \lambda} \frac{\partial}{\partial \lambda} \phi_{\mu, \lambda}^{\prime}-\phi_{-\mu, \lambda}^{\prime} \frac{\partial}{\partial \lambda} \phi_{\mu, \lambda}\right) .
$$

Differentiating the above with respect to $x$ we see that since

$$
\frac{\partial}{\partial x} F_{i}=\int_{\lambda_{i}}^{\lambda} \frac{C\left(x, \lambda^{\prime}\right)}{2 \mu_{0}\left(\lambda^{\prime}\right)} d \lambda^{\prime}
$$

and $\phi_{-\mu, \lambda} \phi_{\mu, \lambda}=C / 2 \mu_{0}$ the right-hand-side of (5.18) is $x$-independent, as it must be. Thus we can solve (5.18) for a function of $\lambda$ only.

We may establish the asymptotics of $f_{i}$ by using the observation of Sect. 3 that $C=p\left(\lambda, T_{j}\right) R(x, \lambda)+\hbar x / 2 \lambda+\mathcal{O}\left(1 / \lambda^{2}\right)$, where $R(x, \lambda)$ is the resolvent of the Schrödinger operator, to find $\mu_{\hbar}=\frac{1}{2}\left[p+\hbar x / z+\mathcal{O}\left(1 / z^{3}\right)\right]$ so that ${ }^{12}$

$$
F_{i}\left(x, \lambda, T_{j}\right)=-\frac{1}{4} \sum_{j} T_{j}^{(1)} z^{2 j+1}+x z+\mathcal{O}(1 / z) .
$$

Note the occurrence of the parameters $T_{j}^{(1)}$. Since $u$ satisfies $\mathrm{KdV}$ flow in $T_{j}$ we know from the standard theory that the BA functions have asymptotics

$$
\phi_{\mu, \lambda}=\frac{1}{\sqrt{2 z}} e^{x z-\frac{1}{4} \sum T_{j}^{(1)} z^{2 \jmath+1}}\left(1+\frac{a_{1}\left(x, T_{j}\right)}{z}+\cdots\right),
$$

from which it follows that $\partial / \partial z\left(\log f_{i}(z)\right)=\mathcal{O}\left(1 / z^{2}\right)$, which completes the proof.

The different solutions $\Psi_{i}$ with WKB asymptotics in the various sectors $\Omega_{i}$ will not agree on the overlaps of sectors because of the Stokes phenomenon at the irregular singular point at infinity. There is further disagreement from the conditions for matching to the well-defined solutions at the turning points $\lambda_{i}$ as we now describe.

For $\left|\delta \lambda_{i}\right|=\left|\lambda-\lambda_{i}\right| \leq \mathcal{O}\left(\hbar^{2 / 3-\varepsilon}\right)$ we can expand

$$
\hbar \frac{\partial \Psi}{\partial \lambda}=\left(\mathscr{A}_{i}+\delta \lambda_{i} \dot{A}_{i}+\mathcal{O}\left(\hbar,\left(\delta \lambda_{i}\right)^{2}\right)\right) \Psi
$$

and the determinant $\mu^{2}=\dot{\mu}_{i}^{2} \delta \lambda_{i}$. Defining a variable $\eta=\hbar^{-2 / 3} \dot{\mu}_{i}^{2 / 3} \delta \lambda_{i}$ and $\tau=\hbar^{1 / 3} \dot{\mu}_{i}^{2 / 3}$, one easily shows that the WKB solution has asymptotics

$$
\left(\frac{C_{i}}{2 \tau}\right)^{1 / 2} \eta^{-1 / 4}\left(\begin{array}{cc}
1 / C_{i} & A_{i} / C_{i} \\
0 & 1
\end{array}\right)\left(\begin{array}{cc}
\tau \eta^{1 / 2} & -\tau \eta^{1 / 2} \\
1 & 1
\end{array}\right) \exp \left[\frac{2 \sigma_{3}}{3} \eta^{3 / 2}\right]
$$

12 From (3.6) we see that we are simply verifying the fact that the asymptotics in $\hbar$ and $\zeta$ is double 
up to right-multiplication by a diagonal constant matrix. On the other hand, after transforming (5.20) by the first matrix in (5.21) we can obtain an exact solution in terms of Airy functions:

$$
\Psi=\left(\begin{array}{cc}
\tau B i^{\prime}(\eta) & \tau A i^{\prime}(\eta) \\
B i(\eta) & A i(\eta)
\end{array}\right)
$$

We can now use the standard asymptotic expansion of these functions [45]:

$$
\begin{aligned}
& A i(\eta) \sim \frac{1}{2 \sqrt{\pi}} \eta^{-1 / 4} e^{-2 \eta^{3 / 2} / 3}\left(1+\mathcal{O}\left(\eta^{-2 / 3}\right)\right),-\pi<\arg \eta<\pi, \\
& B i(\eta) \sim \frac{1}{\sqrt{\pi}} \eta^{-1 / 4} e^{-2 \eta^{3 / 2} / 3}\left(1+\mathcal{O}\left(\eta^{-2 / 3}\right)\right),-\pi / 3<\arg \eta<\pi / 3, \\
& B i(\eta) \sim \frac{1}{\sqrt{\pi}} \eta^{-1 / 4}\left[e^{-2 \eta^{3 / 2} / 3}+\frac{i}{2} e^{-2 \eta^{3 / 2} / 3}\right],-\pi / 3<\arg \eta<\pi, \\
& B i(\eta) \sim \frac{1}{\sqrt{\pi}} \eta^{-1 / 4}\left[e^{-2 \eta^{3 / 2} / 3}-\frac{i}{2} e^{-2 \eta^{3 / 2} / 3}\right],-\pi<\arg \eta<\pi / 3 .
\end{aligned}
$$

We see that to match onto a well-defined framing near $\lambda_{i}$ the standard solutions $\Psi_{i}$ must be multiplied by the nontrivial lower-diagonal matrices

$$
\left(\begin{array}{cc}
1 & 0 \\
\pm i / 2 & 1
\end{array}\right)
$$

Thus, well defined framings lead to fragmented bits of Riemann surface, and well-defined Riemann surfaces lead to discontinuous jumps in framings. This completes the justification of the picture mentioned above.

We expect that the above discussion can be generalized easily to Riemann surfaces which are $n$-fold coverings of the plane. In this case the Schrödinger operator is replaced by the operator $L=D^{n}+\cdots$. One can still define the notion of a BA framing of an $n$-plane bundle on the $\lambda$ line and so on. We also remark that in the above analysis we have occasionally made use of the fact that the point at infinity is a Weierstrass point, but this is probably not essential.

Quantum Moduli Space. As $\hbar \rightarrow 0$, the parameters $T_{i}^{(0)}$ define the moduli of the classical surface $\Sigma_{0}$. On the other hand, if we allow the $T_{i}$ to vary: $T_{i}=$ $T_{i}^{(0)}+\hbar T_{i}^{(1)}+\cdots$, then the $T_{i}^{(1)}$ become coordinates on the Jacobian of $\Sigma_{0}$, as we can see from (2.7) as well as from (5.19). Thus, we propose that one should regard the space of $\left(x, T_{j}\right)$ as a generalization of the universal Jacobian over moduli space. This suggests several interesting questions. For example, is there an analog of the modular group? Can we study limits like $T \rightarrow \infty$ using known results about the boundary of the moduli space of curves? And so forth.

Finally, note that another approach to our problem would be to use noncommutative geometry in the sense of Connes. One might begin with the Heisenberg algebra $[P, \lambda]=\hbar$ and look for interesting ideals which would allow us to define the "ring of functions on the noncommutative Riemann surface", and so on. It would be interesting to see if such ideas are related to the above pictures.

\section{Isomonodromy and Free Fermions}

The isomonodromy problem is very natural from the point of view of conformal field theory. Consider, for example, a conformal block of free Ising fermions in 
the presence of spin fields at positions $a_{i}, \mathscr{F} \sim\left\langle\psi(x) \sigma\left(a_{1}\right) \ldots \sigma\left(a_{n}\right) \psi\left(x_{0}\right)\right\rangle$. As is well-known, $\mathscr{F}$ satisfies a differential equation in $x$ and has monodromy as $x$ is continued around the $a_{i}$. As we move the $a_{i}$ the monodromy representation of the braid group remains invariant. This is isomonodromy, usually described in terms of flatness of a vector bundle over moduli space, in the framework of FriedanShenker modular geometry. In this section we will interpret the isomonodromy problem connected with the string equations in conformal-field-theoretic terms. Our paradigm will be the solution of the Riemann-Hilbert problem for the case of regular singular points given ten years ago by the Kyoto school [46]. We review their construction first, in the light of subsequent developments in CFT. Then we consider the case of irregular singular points. Developing further some work of Miwa [47], we find that the theory of irregular singular points can be included at the expense of the introduction of a new kind of operator. In a way we are making a nontrivial extension of conformal field theory by expanding the class of functions admitted in the theory from analytic functions with algebraic singularities to analytic functions with essential singularities. This is reflected in the need to expand the class of operators from twist operators to star operators.

Regular Singular Points. The basic idea of [46] is that the solution to an $m \times m$ matrix differential equation

$$
\frac{d \Psi}{d z}=A(z) \Psi(z)
$$

may be characterized uniquely by its monodromy properties. More precisely, suppose $A$ has only simple poles at points $a_{v}$ and the residue can be diagonalized to $L_{v}$. Then the matrix $\Psi$ can be uniquely characterized by the requirement that

(i) $\Psi\left(z_{0}\right)=1$,

(ii) $\Psi(z)$ is holomorphic in $z \in \mathbb{P}^{1}-\left\{a_{1}, \ldots, a_{n}\right\}$,

(iii) $\Psi(z)=\hat{\Psi}^{(v)}(z) e^{L_{v} \log \left(z-a_{v}\right)}$ for, $z \cong a_{v}$, where $\hat{\Psi}^{(v)}$ is holomorphic and invertible in a neighborhood of $a_{v}$.

Conversely, any such matrix defines a rational matrix $A=\Psi_{z} \Psi^{-1}$ with at most simple poles. Thus, if one can construct appropriate "twist operators" $\varphi_{i}$ such that the correlation function

$$
\Psi_{\beta \alpha}\left(z_{0} ; z\right)=\left(z_{0}-z\right) \frac{\left\langle\bar{\psi}_{\beta}\left(z_{0}\right) \psi_{\alpha}(z) \varphi_{n}\left(a_{n}\right) \ldots \varphi_{1}\left(a_{1}\right)\right\rangle}{\left\langle\varphi_{n}\left(a_{n}\right) \ldots \varphi_{1}\left(a_{1}\right)\right\rangle}
$$

has the correct monodromy properties, then it must be a solution of (6.1). Thus we have reduced the global Riemann-Hilbert problem to the local problem of finding conformal fields $\varphi_{L}(a)$ with the operator product expansion

$$
\psi_{\alpha}(z) \varphi_{L}(a) \sim\left[\mathcal{O}_{L, \alpha}^{0}(a)+(a-z) \mathcal{O}_{L, \alpha}^{1}(a)+\cdots\right](z-a)^{L_{\alpha}} .
$$

We can construct examples of such $\varphi_{L}$ explicitly via bosonization of the fermions so that:

$$
\begin{aligned}
\psi_{\alpha} & =e^{i \phi_{\alpha}}, \\
\bar{\psi}_{\alpha} & =e^{-i \phi_{\alpha}}, \\
\varphi_{L}(a) & =e^{i \vec{L} \cdot \vec{\phi}},
\end{aligned}
$$

where $\phi_{\alpha}$ are free scalar fields. We now recognize (6.1) as the KnizhnikZamalodchikov equation for $U(1)^{m}$ current algebra, the $\varphi_{L}$ being primary fields associated to a one-dimensional representation of $U(1)^{m}$. 
The construction (6.4) only produces examples with abelian monodromy. More generally, choosing a basis of curves $\gamma_{v}$ circling once around $a_{v}$ and generating the fundamental group $\pi_{1}\left(\mathbb{P}^{1}-\left\{a_{v}\right\} ; z_{0}\right)$ we can construct a differential equation that gives the monodromy $\Psi \rightarrow \Psi M_{v}$ along $\gamma_{v}$ using the operators

$$
\varphi_{M}(a)=\exp \left[\int_{\mathscr{C}}^{a} \operatorname{tr}\{\log (M) J(y)\} \frac{d y}{2 \pi}\right]
$$

where $J_{\beta \alpha}=\bar{\psi}_{\beta} \psi_{\alpha}$ is a $\hat{g} l(m)_{1}$ current and $\mathscr{C}$ is a contour (a branch cut for $\Psi)$ emanating from $a$. Consider now (6.2) with such operators inserted. As we analytically continue $z$ around $a$ the simple pole in the ope of $\psi$ with $J$ gives rise to the monodromy $\psi_{\alpha} \rightarrow \psi_{\gamma} M_{\gamma \alpha}$ in the Fermi field. The $\varphi_{M}(a)$ are again primary fields for a $\hat{U}(1)^{m}$ current algebra, but if $M$ is not diagonal the $U(1)^{m}$ Cartan subalgebra of $g l(m)$ has been rotated. In this case (6.1) is seen to be an interesting generalization of the $\mathrm{KZ}$ equations ${ }^{13}$.

Let us now consider isomonodromic deformation of (6.1). It is clear from locality of the ope that changing the $a_{v}$ leaves the monodromy data unchanged. An old result of Schlesingen states that a necessary and sufficient condition for isomonodromic deformation is

$$
\begin{aligned}
& \frac{\partial \Psi}{\partial z_{0}}=-\sum \frac{A_{v}}{z_{0}-a_{v}} \Psi, \\
& \frac{\partial \Psi}{\partial a_{v}}=\left(-\frac{A_{v}}{z-a_{v}}+-\frac{A_{v}}{z_{0}-a_{v}}\right) \Psi .
\end{aligned}
$$

Taking into account that $\Psi$ is a ratio of correlators we see that (6.6) are again $\mathrm{KZ}$ type equations. The compatibility conditions for these linear quations given the nonlinear Schlesinger equations ${ }^{14}$. The linear equations $(6.1),(6.6)$ associated to the nonlinear isomonodromic deformation equations should therefore be thought of as transport equations on moduli space, so that the theory of isomonodromic deformation for regular singular points fits nicely into the framework of FriedanShenker modular geometry.

According to the general theory of isomonodromic deformation [25-27] there is a tau function associated to the deformation parameters $a_{v}$. In this case it is given by

$$
d \log \tau\left(a_{1}, \ldots, a_{n}\right)=-\sum_{\nu} \operatorname{Res}_{z=a_{v}} \operatorname{tr}\left[\left(\bar{\Psi}^{(v)}\right)^{-1} \frac{\partial \hat{\Psi}^{(\varphi)}}{\partial z} d\left(\log \left(z-a_{v}\right) L_{v}\right)\right]
$$

where the $d$ is a differential in the parameters $a_{v}$. A very interesting result of [25-27] is that this tau function is given by $\tau=\left\langle\varphi_{1} \ldots \varphi_{n}\right\rangle$. We will now rederive this using general principles of conformal field theory.

We have normalized (6.2) so that it is equal to $\delta_{\alpha \beta}$ at $z=z_{0}$. Taking the operator product expansion as $z \rightarrow z_{0}$ and matching this with an expansion of a

13 Technically the standard treatment of Knizhnik-Zamalodchikov equations fails because 1) the $\varphi_{M}$ are not, in general, primary fields for the $\hat{g} l(m)_{1}$ (nor for the $\left.\hat{o}(2 m)_{1}\right)$ current algebra, and 2) one cannot deform the contour integral of $J$ through the branch cut $\mathscr{C}$

14 For an appropriate choice of matrices, for example, these equations reduce to PVI [26] 
solution to (6.1) around $z_{0}$ we find

$$
\begin{aligned}
\frac{\left\langle J_{\beta \alpha}\left(z_{0}\right) \varphi_{n}\left(a_{n}\right) \ldots \varphi_{1}\left(a_{1}\right)\right\rangle}{\left\langle\varphi_{n}\left(a_{n}\right) \ldots \varphi_{1}\left(a_{1}\right)\right\rangle} & =A\left(z_{0}\right)_{\beta \alpha}, \\
\frac{\left\langle T\left(z_{0}\right) \varphi_{n}\left(a_{n}\right) \ldots \varphi_{1}\left(a_{1}\right)\right\rangle}{\left\langle\varphi_{n}\left(a_{n}\right) \ldots \varphi_{1}\left(a_{1}\right)\right\rangle} & =\frac{1}{2} \operatorname{tr} A^{2}\left(z_{0}\right),
\end{aligned}
$$

where $T$ is the stress energy tensor. Since $L_{-1}$ always takes a derivative with respect to position we have

$$
\frac{\partial}{\partial a_{v}} \log \left[\left\langle\varphi\left(a_{1}\right) \ldots \varphi\left(a_{n}\right)\right\rangle\right]=\oint_{a_{v}} d z_{0} \frac{\left\langle T\left(z_{0}\right) \varphi_{n}\left(a_{n}\right) \ldots \varphi_{1}\left(a_{1}\right)\right\rangle}{\left\langle\varphi_{n}\left(a_{n}\right) \ldots \varphi_{1}\left(a_{1}\right)\right\rangle}=\oint_{a_{v}} d z_{0} \frac{1}{2} \operatorname{tr} A^{2}\left(z_{0}\right) .
$$

On the other hand, substituting the local expansion $\Psi=\hat{\Psi}(z-a)^{L}$ we get

$$
\hat{\Psi}^{-1} \hat{\Psi}_{z}+L /(z-a)=\hat{\Psi}^{-1} A \hat{\Psi} .
$$

Squaring this equation we find

$$
\frac{\partial}{\partial a}(\log \tau)=\operatorname{Res} \operatorname{tr}\left(\hat{\Psi}^{-1} \hat{\Psi}_{z} \frac{L}{z-a}\right)=\frac{1}{2} \operatorname{Res} \operatorname{tr} A^{2},
$$

and hence the tau function is simply the correlation function of twist operators.

We note in passing that an easy implication of the above considerations is that the solutions of the stationary $\mathrm{KdV}$ equations themselves can be obtained from an isomonodromy problem [26]. The basic idea is that fermions on a Riemann surface $\sum_{0}$ which is an $n$-fold covering of the plane are equivalent to an $n$-tuplet of fermions on the plane in the presence of appropriate branch points, as is well known in the conformal field theory of orbifolds [48-51]. In particular, the BA framing of the previous section has a QFT representation of the form (6.2).

Irregular Singular Points. Let us now attempt to repeat the previous discussion for the case of a differential equation (6.1), where $A$ is rational but can have irregular singularities. Our treatment is the same in spirit as the discussion of T. Miwa [47], although there are some differences of detail.

Recall the discussion from the beginning of Sect.3. At an irregular singular point we divide up a neighborhood of the point into sectorial domains, each containing a fundamental solution with asymptotics (3.2). In particular, the analytic continuation of $\Psi_{1}$ will have the asymptotic expansion

$$
\Psi_{1} \sim\left[\sum_{l \geq 0} \hat{\Psi}^{(l)}(z-a)^{l}\right](z-a)^{L} e^{T}\left(S_{1} \ldots S_{k-1}\right)^{-1}
$$

in the sector $\Omega_{k}$. A solution to the differential equation can be uniquely characterized by the conditions (i)-(iii) above except that (iii) must be replaced by the requirement that $\Psi$ have the asymptotic expansions (6.11). Thus, to find the solution to an equation with irregular singular points using quantum field theory we define $\tilde{\Psi} \equiv \Psi_{1} e^{-T(z-a)}$ if there is one irregular singular point, and by $\prod_{i} e^{-T\left(z-a_{v}\right)}$ for several irregular singular points, and search for quantum field operators $V_{S, T, L}(a)$, which we call "star" operators, such that

$$
\tilde{\Psi}_{\beta \alpha}\left(z_{0} ; z\right)=\left(z_{0}--z\right) \frac{\left\langle\bar{\psi}_{\beta}\left(z_{0}\right) \psi_{\alpha}(z) V_{S_{1}, T_{1}, L_{1}}\left(a_{1}\right) \ldots\right\rangle}{\left\langle V_{S_{1}, T_{1}, L_{1}}\left(a_{1}\right) \ldots\right\rangle} .
$$


The star operator is characterized by its operator product expansion with $\psi$, $\bar{\psi}$, e.g., for $z \in \Omega_{k}$ we have

$$
\psi_{\alpha}(z) V_{S, T, L}(a) \sim\left[\mathcal{O}_{\gamma}^{0}(a)+(a-z) \mathcal{O}_{\gamma}^{1}(a)+\cdots\right](z-a)^{L_{\gamma}}\left[e^{T}\left(S_{1} \ldots S_{k-1}\right)^{-1} e^{-T}\right]_{\gamma \alpha} .
$$

From this description it looks very unlikely that star operators exist ${ }^{15}$. We now give at least a formal construction of these operators.

Consider a ray $\mathscr{C}$ emanating from a point $a$. Consider the product of operators

$$
\exp \left[\int_{\mathscr{C}}^{a} d y \psi_{\alpha}(y) M_{\alpha \beta}(y) \bar{\psi}_{\beta}(y)\right] \psi_{\alpha}(z),
$$

where $M$ is some matrix defined along the line. We may imagine defining an operator formalism by "angular ordering" (as opposed to the standard radial ordering) in the neighborhood of $a$. If we then analytically continue $z$ in $\psi(z)$ through the curve $\mathscr{C}$ and compare with the other operator ordering it is a simple consequence of Cauchy's theorem and the operator product expansion that we have the exchange algebra:

$$
\begin{aligned}
& \exp \left[\int_{\mathscr{C}}^{a} d y \psi_{\alpha}(y) M_{\alpha \beta}(y) \bar{\psi}_{\beta}(y)\right] \psi_{\alpha}(z-\varepsilon) \\
& =\psi_{\gamma}(z+\varepsilon)\left(e^{M(z)}\right)_{\gamma \alpha} \exp \left[\int_{\mathscr{C}}^{a} d y \psi_{\alpha}(y) M_{\alpha \beta}(y) \bar{\psi}_{\beta}(y)\right],
\end{aligned}
$$

where $z$ is a point on $\mathscr{C}$ and $z \pm \varepsilon$ are points above and below $z$. Thus, defining $\mathscr{S}_{k} \equiv e^{T} S_{k} e^{-T}$ we may define, at least formally,

$$
V_{S, T, L}(a)=\varphi_{L}(a) \prod_{k} \exp \left[\int_{\mathscr{C}_{k}}^{a} \operatorname{tr}\left\{\left(\log \mathscr{S}_{l}(y)\right) J(y)\right\} d y\right]
$$

where we choose contours $\mathscr{C}_{k}$ in $\Omega_{k}$ such that the matrix $\mathscr{S}_{k}$ approaches the identity rapidly.

It follows from locality of the operator product expansion that the differential equation satisfied by $(6.12)$ has the property that the monodromy data $S$, $L$ are preserved if we vary the parameters $a_{i}, T_{i}$. Just as the tau function for isomonodromic deformation of an equation with regular singular points is given by the correlation function of twist operators, the tau function for isomonodromic deformation in an equation with irregular singular points is given by the correlation function of star operators [47]. We can prove this as follows.

\footnotetext{
15 For example, it is often claimed that operator product expansions in CFT are convergent. Note that (6.13) is only asymptotic. The reason for this is ultimately to be found in the fact that the string coupling has become dimensionful [2]. Note that it is $x$ and the masses $T_{j}$ which multiply the terms giving the essential singularity at infinity. In this context it is interesting to note that many integrable massive field theories have correlation functions related to Painlevé equations [16, 27, 52]
} 
First consider the dependence on the position $a$. By an argument analogous to the twist operator case we have

$$
\begin{aligned}
& \frac{\left\langle J_{\beta \alpha}\left(z_{0}\right) V_{S_{1}, T_{1}, L_{1}}\left(a_{1}\right) \ldots\right\rangle}{\left\langle V_{S_{1}, T_{1}, L_{1}}\left(a_{1}\right) \ldots\right\rangle}=-A\left(z_{0}\right)+T^{\prime}\left(z_{0}\right), \\
& \frac{\left\langle T\left(z_{0}\right) V_{S_{1}, T_{1}, L_{1}}\left(a_{1}\right) \ldots\right\rangle}{\left\langle V_{S_{1}, T_{1}, L_{1}}\left(a_{1}\right) \ldots\right\rangle}=\frac{1}{2} \operatorname{tr}\left(A-T^{\prime}\right)^{2}\left(z_{0}\right) .
\end{aligned}
$$

Taking the residue of the ope with the stress tensor gives the derivative of $V_{S, T, L}(a)$ with respect to position $a$. However, $V$ also depends on $a$ through the data $T(z-a)$ and we must take this into account. Commutation with $J$ rotates the fermions and hence rotates the fermion transition functions in the adjoint representation. Since a change in $T$ induces a change $\delta \mathscr{S}=[\delta T, \mathscr{S}]$ we find that

$$
\operatorname{tr}\left(\delta T\left(z_{0}\right) J\left(z_{0}\right)\right) V_{S, T, L}(a) \sim \int_{\mathscr{C}}^{a} \frac{d y}{z_{0}-y} \operatorname{tr}\left(\delta T\left(z_{0}\right) \frac{\delta}{\delta T(y)}\right) V_{S, T, L} .
$$

Using (6.16) and (6.17) we get

$$
-\operatorname{Res}_{z_{0}=a} \operatorname{tr} \delta T\left(z_{0}\right) A\left(z_{0}\right)=\frac{\sum_{k} \int_{\mathscr{C}_{k}}^{a} d y \operatorname{tr}\left(\delta T(y) \frac{\delta}{\delta T(y)}\right)\left\langle V_{S, T, L} \ldots\right\rangle}{\left\langle V_{S, T, L}(a) \ldots\right\rangle} .
$$

Putting together (6.16) and (6.18) we then find

$$
\frac{d}{d a} \log \langle V \ldots V\rangle=\frac{1}{2} \operatorname{Res}_{z_{0}=a} \operatorname{tr} A^{2}\left(z_{0}\right)=\frac{d}{d a} \log \tau,
$$

where the second equality follows from an argument similar to the case of regular singularities. It also follows immediately from (6.18) that the dependence of $\log \tau$ and $\log \langle V \ldots V\rangle$ on other parameters is the same.

In [47] Miwa obtained formulae for the tau function using a slightly different formalism. Comparing his formulae in terms of infinite expansions evaluated by Wick's theorem we obtain the same result:

$$
\tau=\sum_{l=0}^{\infty} \frac{(-1)^{l}}{l !} \sum_{\alpha_{l}=1}^{m} \int d x_{1} \ldots d x_{l} \operatorname{det}\left[\frac{1}{2 \pi i} \frac{1}{x_{j}-x_{k}} \frac{\left(e^{T\left(x_{j}\right)}\right)_{\alpha_{j}}}{\left(e^{T\left(x_{k}\right)}\right)_{\alpha_{k}}} \log S_{\alpha_{j} \alpha_{k}}\right] .
$$

As shown in [47] contours of integration can be defined so that for small enough Stokes data the integrals make sense, thus giving a more precise definition to the star operator.

As a special case of the above formalism we can express the solution $u$ the string equations in terms of a fermion correlation function. For the $(2 l-1,2)$ models we have a star operator at infinity and a twist operator at the origin. We may choose the contours of the star operator to converge to the branch cut for the twist operator at the origin. Then applying (6.18) to the case where we vary the parameter $x$, (one of the parameters in $T(\zeta)$ ) we see that the tau function for 


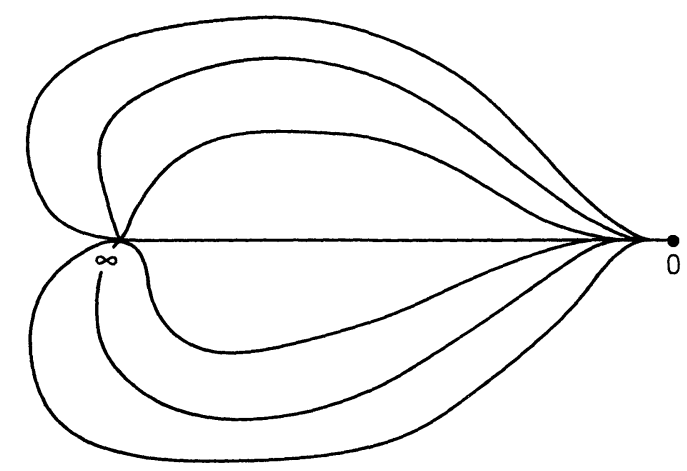

Fig. 4. A schematic view of a choice of contours for the star operator

the string equations can be computed using the star operator,

$$
\begin{aligned}
V_{S, x}(a)= & e^{i\left(\phi_{1}(a)+\phi_{2}(a)\right) / 2} \prod_{k} \exp \left[s_{2 k+1} \int_{\mathscr{C}_{2 k+1}}^{a} e^{2 T(y)} \psi_{1} \bar{\psi}_{2}(y)\right] \\
& \times \exp \left[s_{2 k} \int_{\mathscr{C}_{2 k}}^{a} e^{-2 T(y)} \psi_{2} \bar{\psi}_{1}(y)\right],
\end{aligned}
$$

where a choice of contours is shown in Fig. 4, and a twist operator $\varphi$, as the two-point function:

$$
\tau(x)=\left\langle V_{S, x}(\infty) \varphi(0)\right\rangle .
$$

From this construction it appears that the correlation functions of twist operators always provide a solution of the inverse monodromy problem and the corresponding string equation. The only thing which can go wrong is that the correlation function, as a function of $x$ might have a zero, so that $u$ has a second order pole.

As in the case of regular singularities we may interpret the equations of (2.7) as transport equations. Following the interpretation of Sect. 5 we see that these should be called transport equations on quantum moduli space. The discussion of Sect. 5 must have a parellel discussion here. In particular, since the $\hbar \rightarrow 0$ limit of a quantum Ba framing gives a standard BA framing, the "classical limit" of a star operator must be a product of twist operators. It would be very interesting to see in detail how this limit is obtained.

Grassmannians and Determinants. It is well known that in the case of almost periodic solutions to the $\mathrm{KdV}$ hierarchy the tau function is properly thought of as a section of a determinant line bundle [13] and that this determinant line bundle is just the vacuum bundle for free fermions defined on the algebraic curve associated to the solution $u(x)$ via the BCK theory. We can argue that, with some modifications, this picture continues to hold for the tau functions associated to the isomonodromy problems discussed above.

Recall that in the operator formalism we choose a disc surrounding some point $P$ and define a local Hilbert space on a circle surrounding that point. The 
partition function of fermions on the surface may be regarded as a function of the line bundle $\mathscr{L} \rightarrow \Sigma-\{P\}$ of which the fermion wavefunctions are holomorphic sections. By considering the restriction of these sections to a circle surrounding $P$ we obtain a subspace $W \subset L^{2}\left(S^{1}\right)$ defining an element of the Grassmannian. Denoting by $\Omega$ the fermion vacuum created by the geometry $\mathscr{L} \rightarrow \Sigma-\{P\}$, that is, $\Lambda^{\mathrm{max}} W$ we obtain:

$$
\tau_{W}=\left\langle 0 \mid \Omega_{W}\right\rangle=\int_{W} d \psi d \bar{\psi} e^{\int \bar{\psi} \bar{\partial} \psi}=\operatorname{Det} \bar{\partial}_{W} .
$$

As explained in [53] we may easily incorporate correlation functions into this picture through use of the multiplicative Ward identities.

Recall that if we want a correlator of $\left\langle\prod \psi\left(P_{i}\right) \prod \bar{\psi}\left(Q_{i}\right)\right\rangle$ when the fermion field lives in the line bundle $\mathscr{L} \rightarrow \Sigma$ associated to $W \in \mathrm{Gr}$ we proceed as follows. Note that the Lagrangian is invariant under $\psi \rightarrow f \psi, \bar{\psi} \rightarrow f^{-1} \bar{\psi}$. This does not imply that the expression (6.22) is invariant, since the allowed space of sections is drastically changed. First of all, the boundary conditions are modified by $W \rightarrow f \cdot W$. Secondly, at zeroes and poles of $f$ the $L^{2}$ condition on the wavefunctions becomes an unusual normalizability constraint. Using a local analysis of the Hilbert space on a circle surrounding such a pole one can show [53] that this unusual constraint can be replaced by the insertion of an appropriate operator in the presence of ordinary fermions. As an example, let us take $f$ to have a single zero and pole. Taking into account the conformal weight one half of fermions we get

$$
\left[\left(d f^{-1} / d z\right)_{P}(d f / d z)_{Q}\right]^{-1 / 2}\langle\bar{\psi}(P) \psi(Q)\rangle_{f \cdot W}=\frac{\operatorname{Det} \bar{\partial}_{W}}{\operatorname{Det} \bar{\partial}_{f \cdot W}} .
$$

As pointed out in [53] this is the physical explanation for the relation between the Baker function and the tau function.

Suppose now we have an $m$-tuplet of fermions $\psi_{\alpha}$ defined on a Riemann surface $\Sigma$. Removing a neighborhood of a point $P \in \Sigma$, the boundary conditions of $\vec{\psi}$ define a subspace of $W \subset \operatorname{Gr}\left(H^{m}\right)$, where $H=L^{2}\left(S^{1}\right)$ as usual ${ }^{16}$. The partition function is again Det $\bar{\partial}_{W}$ as usual, and the bottom row of the BakerAkhiezer framing describes a holomorphic section of a holomorphic $m$-plane bundle over $\Sigma-P$.

The theory of multiplicative Ward identities again holds, but with some interesting generalizations, the transformations:

$$
\begin{aligned}
& \vec{\psi}(\zeta) \rightarrow Y(\zeta) \vec{\psi}(\zeta), \\
& \overrightarrow{\bar{\psi}}(\zeta) \rightarrow\left(Y^{-1}\right)^{\operatorname{tr}}(\zeta) \overrightarrow{\bar{\psi}}(\zeta)
\end{aligned}
$$

preserve the action. The multiplication by $Y$ will again change the boundary conditions (by the obvious multiplicative action of $Y(\zeta)$ on $H_{m}$ ) and if $Y$ has singularities then the $L^{2}$ condition on the fermi wavefunctions is modified in the

\footnotetext{
16 The relation between the quantum field theory of a fermion on a surface $\Sigma$ which is an $m$-fold cover of the plane, and the quantum field theory of an $m$-tuplet of fermions on the plane in the presence of branch points gives a nice explanation of the construction on pp. 34-35 of [13]. Moreover, it shows directly why their association of a space $W$ in $\mathrm{Gr}^{(n)}$ to an $n^{\text {th }}$ order operator coincides with the space constructed via the BCK theory in the case that the operator satisfies the stationary generalized $\mathrm{KdV}$ equations
} 
neighborhood of these singularities. Again, we can replace these conditions by operator insertions. If $Y$ has poles or zeroes then the operators are simply products of $\psi_{\zeta}, \bar{\psi}_{\alpha}$ and their derivatives. If $Y$ has branch cuts then we have inserted twist operators $\varphi_{L}$. A rigorous discussion of construction of the associated $\bar{\partial}$ operator and its determinant for the insertion of twist operators on $\mathbb{P}^{1}$ has been given in [54]. Finally, if $Y$ has essential singularities associated to an irregular singular point of a differential equation then we have inserted a star operator. In all cases we have

$$
\langle V \ldots\rangle_{W} \sim \frac{\operatorname{Det} \bar{\partial}_{Y^{-1} \cdot W}}{\operatorname{Det} \bar{\partial}_{W}}
$$

Moreover, and quite generally, if we consider the multiplicative WI's for a correlation function of twist and star operators obtained by multiplying the Fermi field by a function with a single zero and pole we obtain a "discrete" relation between the fermion two point function in the presence of these operators and a ratio of tau functions. We expect that one can explain in conformal-fieldtheoretic terms all the relations obtained by use of "Schlesinger transformations" in [26]. (Schlesinger transformations themselves can be viewed as replacing a twist operator by its normal ordered product with $\psi, \bar{\psi}$.)

From these remarks we see that the KdV flow is again induced by the infinite dimensional abelian group $\Gamma_{+}$of [13]. In this case the flow has the interesting interpretation of being the flow of the renormalization group.

\section{Free Fermions from Matrix Models}

In this section we suggest a way in which the connections between free-fermion theory and the string equations discussed above might be derivable directly from the matrix model integrals. Unfortunately our results are incomplete, but some key features emerge already at this stage. First we will see that the spectral parameter of inverse scattering theory is identified with the eigenvalue coordinate of the random matrix path integral. Second we will see that the random matrix model leads naturally to a doublet of fermions.

Let us consider the simple Gaussian matrix model.

$$
\begin{aligned}
Z_{N} & =\int D^{N^{2}} \phi e^{-\frac{1}{2} N \operatorname{tr} \phi^{2}} \\
& =\int \prod d \lambda_{i} \Delta^{2} e^{-\frac{1}{2} N} \sum_{i} \lambda_{i}^{2}
\end{aligned}
$$

Correlation functions of, say, traces of $\phi^{n}$ can be interpreted $[6,55,56]$ as expectation values in a slater determinant of fermion one-body wavefunctions given by Hermite functions:

$$
\psi_{j}(\lambda)=\left(\frac{N}{2 \pi}\right)^{1 / 4}\left(\frac{N}{j !}\right)^{1 / 2}\left(\lambda^{j}+\cdots\right) e^{-N \lambda^{2} / 4}
$$


Fig. 5. A simple Fermi sea

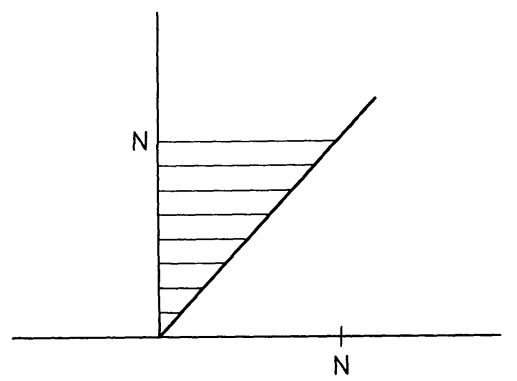

As in [6] we may pass to second quantized wavefunctions:

$$
\begin{aligned}
\psi(\lambda) & =\sum_{n=1}^{\infty} \psi_{n}(\lambda) a_{n}, \\
\psi(\lambda)^{\dagger} & =\sum_{n=1}^{\infty} \psi_{n}(\lambda) a_{n}^{\dagger}, \\
\left\{\psi^{\dagger}(\lambda), \psi\left(\lambda^{\prime}\right)\right\} & =\delta\left(\lambda-\lambda^{\prime}\right) .
\end{aligned}
$$

The Fermi sea is illustrated in Fig. 5. As argued in [6] the main contributions to correlation functions come from the neighborhood of the Fermi level. In the large $N$ limit, and for $n \sim \mathcal{O}(N)$ we have:

$$
\begin{aligned}
\psi_{2 n}(\gamma / N) & \sim(-1)^{n}\left(\frac{N}{2 \pi^{2} n}\right)^{1 / 4} \cos \left(\gamma \sqrt{\frac{(4 n+1)}{2 N}}\right), \\
\psi_{2 n+1}(\gamma / N) & \sim(-1)^{n}\left(\frac{N}{2 \pi^{2} n}\right)^{1 / 4} \sin \left(\gamma \sqrt{\frac{(4 n+3)}{2 N}}\right) .
\end{aligned}
$$

Thus, defining $\hat{\psi}(\gamma ; N) \equiv \frac{1}{\sqrt{N}} \psi(\gamma / N)$ we argue that $\hat{\psi}$ actually has a smooth large $N$ limit. For example, for the two-point function one easily verifies:

$$
\left\langle N\left|\hat{\psi}^{\dagger}(\gamma) \hat{\psi}\left(\gamma^{\prime}\right)\right| N\right\rangle \rightarrow \frac{1}{\pi} \frac{\sin \left(\gamma-\gamma^{\prime}\right.}{\gamma-\gamma^{\prime}}
$$

On the operator level we define $p=n / 2 N$ and

$$
\begin{aligned}
& a_{1}(p)=\sqrt{N}(-1)^{n+N}\left(\hat{a}_{2 n}-i \hat{a}_{2 n+1}\right), \\
& a_{2}(p)=\sqrt{N}(-1)^{n+N}\left(\hat{a}_{2 n}-i \hat{a}_{2 n+1}\right),
\end{aligned}
$$

where $\hat{a}_{n} \equiv a_{N+n}$. The sum over $n$ becomes an integral $\int_{-1}^{\infty} d p$. Again, assuming that the main contributions come from the neighborhood of the Fermi level we extend this to an integral over the entire $p$ axis. Our main claim is therefore that 
$\hat{\psi}$ has a good large $N$ limit and is given by

$$
\begin{aligned}
\hat{\psi}(\gamma) & =e^{i \gamma} \int_{-\infty}^{\infty} d p a_{1}(p) e^{i \gamma p}+e^{-i \gamma} \int_{-\infty}^{\infty} d p a_{2}(p) e^{-i \gamma p} \\
& =e^{i \gamma} \psi_{1}(\gamma)+e^{-i \gamma} \psi_{2}(-\gamma)
\end{aligned}
$$

and that the Fermi sea becomes the ground state defined by $a_{i}(p)|o\rangle=0$ for $p>0$ and $a_{i}^{\dagger}(-p)|0\rangle$ for $p>0$. This will only be true in correlation functions where the delta function singularity between $\hat{\psi}$ and $\hat{\psi}^{\dagger}$ does not play an important role.

Level Spacing. As a test of these ideas we rederive a formula for the level spacing in the gaussian unitary ensemble from the theory of random matrices. We are simply reinterpreting the calculation of Jimbo et al. [57]. The point of this exercise is that in this case we can translate directly from a random matrix partition function to a tau function in a free fermion system with $g l(2)$ symmetry.

In the model (7.1) we ask for the probability that no eigenvalue falls in the range $\left[\lambda_{1}, \lambda_{2}\right] .{ }^{17}$ In terms of the orthogonal polynomials $\psi_{j}$ one may show this quantity to be given by $[55,56]$

$$
\begin{aligned}
\Delta_{I} & =\operatorname{det}\left[\delta_{j, k}-\int_{\lambda_{1}}^{\lambda_{2}} d \lambda \psi_{j}(\lambda) \psi_{k}(\lambda)\right]_{0 \leq j, k \leq N-1} \\
& =\left\langle N\left|\exp \left(-\int_{\lambda_{1}}^{\lambda_{2}} \hat{\psi}^{\dagger}(\lambda) \hat{\psi}(\lambda) d \lambda\right)\right| N\right\rangle .
\end{aligned}
$$

In the $N \rightarrow \infty$ limit, taking $\lambda_{i}=a_{i} / N$, we obtain

$$
\Delta_{I}(\xi)=\left\langle 0\left|: \exp \left(-\pi \xi \int_{I} \hat{\psi}^{\dagger} \hat{\psi}\right):\right| 0\right\rangle
$$

for $\xi=1 / \pi$ and $I=\left[a_{1}, a_{2}\right]$. It is understood that the normal-ordered exponential is evaluated by expanding in power series and point-splitting all the integrals so that the delta functions in the two-point correlation functions don't contribute. This is a rather mild version of the star operator. Following [57] we show that $\Delta_{I}$ is simply the $\tau$ function for isomonodromic deformation associated with $g l(2)$ fermions $\psi_{1,2}$, as in the previous sections. Thus we define $\chi_{1}(z)=e^{i z} \psi_{1}(z)$ and $\chi_{2}(z)=e^{i z} \psi_{2}(-z)$ so that $\hat{\psi}=\chi_{1}+\chi_{2}$ and $\psi^{\dagger}=\chi_{1}^{\dagger}+\chi_{2}^{\dagger}$, and consider the Baker-Akhiezer framing.

$$
\Psi_{\alpha \beta}\left(x, x^{\prime}\right)=\frac{\left\langle 0\left|\chi_{\alpha}^{\dagger}(x) \exp \left(-\pi \xi \int_{I} \xi^{\dagger} \chi\right) \chi_{\beta}\left(x^{\prime}\right)\right| 0\right\rangle}{\left\langle 0\left|\exp \left(-\pi \xi \int_{I} \chi^{\dagger} \chi\right)\right| 0\right\rangle}
$$

At this point one may easily follow all the steps in Sect. 7 of [57] using the interpretation in terms of a doublet of fermions ${ }^{18}$. In particular, we can compute the

\footnotetext{
17 This is the same as "the probability of empty place" in [16]

${ }^{18}$ For readers of [57] one can identify, for example, $R_{I}^{ \pm}\left(x, x^{\prime} ; \xi\right)$ with the two point function of $\hat{\chi}_{i}^{\dagger}(x)$ with $\hat{\psi}\left(x^{\prime}\right)$, and so on
} 
monodromy of (7.8) under analytic continuation of $x$ around $a_{i}$. From the operator product expansion we compute $d \Psi \Psi^{-1}$, where $d$ is exterior differentiation in $x, a_{i}$. This gives once more Knizhnik-Zamalodchikov type equations, interpreted here as isomonodromic deformation equations. From the general arguments of the previous section we deduce that $\Delta_{I}$ is the tau function for isomonodromic deformation.

If we consider a more general polynomial potential than (7.1) we must work with the orthonormal wavefunctions $p_{n}(\lambda)$ appropriate to that potential in order to justify the assumption that the important contributions to the correlators of $\hat{\psi}$ come from the neighborhood of the Fermi level. For even potentials we can rewrite the recursion relation for orthogonal polynomials [1-3] in the form:

$$
\begin{aligned}
\lambda p_{2 n}(\lambda) & =\sqrt{r_{2 n+1}} p_{2 n+1}+\sqrt{r_{2 n}} p_{2 n-1}, \\
\lambda p_{2 n+1}(\lambda) & =\sqrt{r_{2 n+2}} p_{2 n+2}+\sqrt{r_{2 n+1}} p_{2 n} .
\end{aligned}
$$

By evaluating these at $\lambda=0$ we see that quite generally if we expect a continuum limit for the orthonormal wavefunctions themselves in the neighborhood of $\lambda=0$ we should define

$$
p_{2 n+1}\left(\frac{\lambda}{N}\right)=(-1)^{n} f_{1}(x, \lambda), \quad p_{2 n}\left(\frac{\lambda}{N}\right)=(-1)^{n} f_{2}(x, \lambda)
$$

where $x=n / N$. Assuming $r_{n}$ has an expansion of the form $r_{n}=r(x)+\varepsilon^{2} r_{1}(x)+\cdots$, where $\varepsilon=1 / N$ we find that (7.9) implies

$$
\begin{aligned}
& f_{1}=(r(x))^{-1 / 4} \sin \left[\lambda \int^{x} \frac{d x^{\prime}}{\sqrt{r\left(x^{\prime}\right)}}\right], \\
& f_{2}=(r(x))^{-1 / 4} \cos \left[\lambda \int^{x} \frac{d x^{\prime}}{\sqrt{r\left(x^{\prime}\right)}}\right] .
\end{aligned}
$$

Since the dominant contributions of physical quantities come from the neighborhood of the Fermi level $(x=1)$ we see that quite generally the orthonormal wavefunctions become sines and cosines leading to a doublet of Fermi fields as above. In particular, at finite $N$ we have the exact formula:

$$
\left\langle N\left|\psi^{\dagger}\left(\lambda_{1}\right) \psi\left(\lambda_{2}\right)\right| N\right\rangle=\sqrt{r_{N+1}} \frac{\psi_{N+1}\left(\lambda_{1}\right) \psi_{N}\left(\lambda_{2}\right)-\psi_{N+1}\left(\lambda_{2}\right) \psi_{N}\left(\lambda_{1}\right)}{\lambda_{1}-\lambda_{2}},
$$

and (7.4) again holds so the discussion from (7.3) to (7.8) may be repeated. From these remarks it follows that the level spacing should be universal.

$2 D$ Gravity. We would like to apply these remarks to $2 D$ gravity. At finite $N$ one can write a Fredholm integral equation for the two-point function, such that the partition function is the Fredholm determinant, giving discrete versions of the BA framing and the tau function. For small $\lambda$ the kernels have a smooth $N \rightarrow \infty$ limit. Unfortunately, exploring the double scaling limit uncovered in [1-3] requires a more delicate analysis of the behavior of the orthogonal polynomials for $\lambda$ near the boundary of the support of the eigenvalue distribution ${ }^{19}$. Nevertheless, we

19 Our difficulty is that different scaling assumptions lead to different asymptotic expressions. For example, assuming the continuum limit $\mathscr{P}_{n}\left(\lambda_{c}+a^{2 / m} \lambda\right) \rightarrow P(z, \lambda)$ we see from the recursion relation that $P(z, \lambda)$ would become the Baker-Akhiezer function. But there are other possibilities 
expect that the effect of the multicritical action will be equivalent to an insertion of a fermion bilinear

$$
\exp \left[\int_{-\infty}^{\infty} d \gamma V(\gamma) \hat{\psi}^{\dagger} \hat{\psi}\right]
$$

which will become the star operator. This interpretation is supported by the fact that the Stokes data for the physical solutions to the string equations are concentrated along the real and imaginary $\zeta$ axis, as shown in Appendix A. (Recall that $\gamma=\zeta^{2}$.) Whether such an interpretation is really tenable must be left to future work. It would give a complete explanation of the relation between the one-matrix model formulation of $2 D$ quantum gravity and the theory of free fermions in the presence of star operators.

According to [5] and [18] $2 D$ gravity coupled to $(p, q)$ minimal models should have $s l(p)$ or $s l(q)$ symmetry. In particular, we expect that an analysis similar to that above should show that in the matrix chain case $s l(n)$ fermions can arise. This would happen if in the recursion relations:

$$
\lambda(1) f_{m}(\lambda(1))=Q_{m, k} f_{k}(\lambda(1))
$$

the orthogonal polynomials $f_{k}$ have different smooth limits for the different conjugacy classes of $k \bmod n$.

\section{Further Speculations}

Since all known matrix models are described by nonlinear equations associated to isomonodromic deformation, one might wonder if this is not a general feature. Possibly $2 D$ gravity should be formulated as a conformal field theory (on auxiliary Riemann surfaces!) in the presence of a new class of operators. This raises many questions. The Riemann surfaces discussed in this paper have no obvious connection to the world sheet of the $2 D$ gravity model. The latter emerges from the Feynman diagram expansion of a theory of a Hermitian matrix $\phi_{i j}$. The surfaces in this paper are coordinatized by the spectral parameter $\lambda$, interpreted in terms of the eigenvalues of $\phi_{i j}$. Nevertheless, it is difficult to believe that the surfaces are unrelated. Indeed, it was suggested by A. Morozov and S. Shatashvili that $2 D$ gravity should be thought of in terms of a nonconformal field theory at genus zero, the effects of the sum over topologies effectively spoiling the conformal invariance [58]. We have arrived at a picture strikingly similar to their guess. Possibly the auxiliary surface is, in some sense, a picture of an effective world-sheet, with the star operator reproducing the effect of a "condensation of handles." (This would raise the spectre of whether we should consider the role of nontrivial topologies of the auxiliary Riemann surface itself.) Continuing this line of thought, it is conceivable that extending the above picture to conformal field theories other than those of free fermions might correspond to exotic types of gravity, or perhaps to $c>1$ theories. For example we can consider star operators in WZW theories. As we saw, the isomonodromic deformation equations can be interpreted as $\mathrm{KZ}$ equations, but these equations were at level 1 . It would be interesting to write down and study the higher level equations. The free field realizations of WZW might facilitate the construction of star-like operators in these models. 
It would also be very interesting to understand if there is a connection between the above ideas and the topological field theory approach of [7-10]. In the latter approach the topological origin of $\mathrm{KdV}$ flows has proven elusive. In the present approach they are interpreted as flows defined by Knizhnik-Zamalodchikov-type equations on a certain moduli space. Perhaps some kind of asymptotic expansion of a star operator in negative powers of $x$ will provide the missing link between these formalisms.

$\mathrm{Be}$ all this as it may, our work has implications beyond the framework of $2 D$ gravity. We have been led to a generalization of modular geometry. Firstly, one should include the notion of star operators in general conformal field theories. Thus, the basic monodromy data of such a theory will include not only matrices representing the braid group and operator product expansions (" $B$ and $F$ matrices") but also Stokes matrices occurring in more exotic exchange algebras than have been hitherto examined. We might therefore expect that these monodromy data will define some generalization of a modular tensor category [59]. Secondly, as we have described, the introduction of star operators leads to a notion of quantum Riemann surface and quantum moduli space. Just as there are analytical and topological aspects to modular geometry (the latter corresponding to the modular functor point of view), one might expect that the analytic aspects associated to this generalized MTC would be related to some geometry of quantum moduli space. The geometric category representing this extended notion of a MTC might well be useful in understanding the analog of modular geometry for integrable but nonconformal models. From what we have managed to glean of the geometrical meaning of this extension we may expect to find a rich but peculiar combination of algebraic and analytic geometry.

\section{Appendix A. An Application: The BMP Solutions}

Recently Brezin, Marinari, and Parisi have proposed that in isolating critical behavior of matrix models one should not take the continuum limit after analytic continuation in couplings, but only with integrals which are initially well-defined [60]. This led them to construct numerically a solution of $R_{3}[u(x)]=x$ which, they argued, is uniquely determined by reality and the asymptotics $u \sim \mathcal{O}\left(x^{1 / 3}\right)$ as $x \rightarrow \pm \infty$. From the numerical integration it appears that the solution is pole-free. It is natural to suppose that such unique solutions for all odd $m$. This proposal for fixing the "nonperturbative parameters" of the string equation has been given further support in $[33,61]$. In this section we will see how the isomonodromic deformation method can give some insights into the results of $[60,61]$.

We will show that the above asymptotics uniquely determines the Stokes parameters in the monodromy problem associated to $R_{3}=x$. This unique solution is real for all $x$. A technicality prevents an easy discussion of the equations for all odd $m$, but we can present a natural conjecture for the behavior of the Stokes matrices in all cases. For all odd $m$ we expect the solution to the unique. Using the property that $\mathrm{KdV}$ flow in $T_{j}$ is isomonodromic we can also confirm the numerical results of [61] that it is impossible to flow from the $m=3$ model to the $m=2$ model. We also will indicate a line of argument which can probably show that these unique solutions have no poles on the real axis.

We investigate the direct monodromy problem following closely the treatment in $[30,62]$. Consider the equation $\mathbb{P}_{l} \Psi=0$ as $x \rightarrow \pm \infty$. We must distinguish 
several cases. Since $R_{l}=\kappa_{l} u^{l}+\cdots$ with

$$
\kappa_{l}=(-1)^{l} \frac{(2 l-1) ! !}{2^{l+1} l !}
$$

we see that perturbation theory predicts that the solution to ${ }^{20} R_{l+1}=-\frac{1}{2} x$ is given by

$$
\begin{array}{lll}
u \sim\left(-1 / 2 \kappa_{l+1}\right)^{1 /(l+1)} x^{1 /(l+1)} & l \text { even, } & x \rightarrow \pm \infty \\
u \sim \pm\left(1 / 2 \kappa_{l+1}\right)^{1 /(l+1)}(-x)^{1 /(l+1)} & l \text { odd, } & x \rightarrow-\infty
\end{array}
$$

In fact, perturbation theory tells us to take the + root for $u$ in the case of odd $l$, but we may easily examine both cases at once. Rescaling variables and only keeping leading order terms we may rewrite the equation $\mathbb{P}_{l} \Psi=0$ as

$$
\frac{d W}{d \xi}=\tau\left[\left(2 \xi^{2} \pm 1\right) p_{l}^{ \pm}(\xi) \sigma_{3} \mp p_{l}^{ \pm}(\xi) i \sigma_{2}-\frac{1}{2 \xi \tau}\left(\frac{8 l \kappa_{l+1}}{l+1} \xi^{2} p_{l}(\xi)+1\right) \sigma_{1}+\mathcal{O}\left(1 / \tau^{2}\right)\right] W,
$$

where $p_{l}^{ \pm}(\xi)=\sum_{p=0}^{l}( \pm 1)^{p} \kappa_{p} \xi^{2 l-2 p}$. For $l$ odd we obtain this equation with $x \rightarrow-\infty$, where:

$$
\zeta=\left(-x / 2 \kappa_{l+1}\right)^{1 /(2 l+2)} \xi, \quad \tau=\left(-x / 2 \kappa_{l+1}\right)^{(2 l+3) /(2 l+2)} .
$$

For $l$ even we obtain this equation with $\pm x \rightarrow+\infty$, where

$$
\zeta=\left( \pm x /\left(-x / 2 \kappa_{l+1}\right)\right)^{1 /(2 l+2)} \xi, \quad \tau=\left( \pm x /\left(-2 \kappa_{l+1}\right)\right)^{(2 l+3) /(2 l+2)} .
$$

The evaluation of the Stokes matrices is carried out by doing a WKB analysis in the $\tau \rightarrow \infty$ limit as in [29]. Thus one obtains true solutions to (A.2) which are asymptotic as $\tau \rightarrow \infty$ to the WKB ansatz:

$$
\left.W^{\mathrm{WKB}} \sim T \exp \tau \int^{\zeta} \Lambda\left(\zeta^{\prime}\right) d \zeta^{\prime}\right]
$$

where $T$ diagonalizes (A.2) to $\Lambda=\mu \sigma_{3}$. There are several WKB solutions in different regions defined by the turning points and conjugate Stokes lines. The turning points are simply the roots $\xi_{i}$ of $\mu$, and the conjugate Stokes lines are the lines defined by the vanishing real part:

$$
\mathfrak{R} \int_{\xi_{\imath}}^{\xi} \mu\left(\xi^{\prime}\right) d \xi^{\prime}=0
$$

For WKB solutions defined in "large" regions abutting an open neighborhood of infinity we can define a connection matrix, $W_{l}^{\mathrm{WKB}}=W_{k} C_{k, l}$ to the corresponding fundamental solution with standard asymptotics in the region $\Omega_{k}$ at infinity. Then by matching the WKB solutions to a well-defined single valued solution $\breve{W}_{i}$ near a turning point $\xi_{i}$, i.e., $W_{l}^{\mathrm{WKB}}=\breve{W}_{i} N_{l, i}$ we can determine the Stokes matrices in terms of the matrices $C, N$. For further details see the book [29].

Our main observation regarding the WKB analysis of (A.2) is the following. Suppose that at a turning point which is a root of $p_{l}$ the conjugate Stokes lines form three large regions each abutting an open region infinity as in Fig. 6 . We

$\overline{20 \text { We set } \hbar=1}$ in this section 


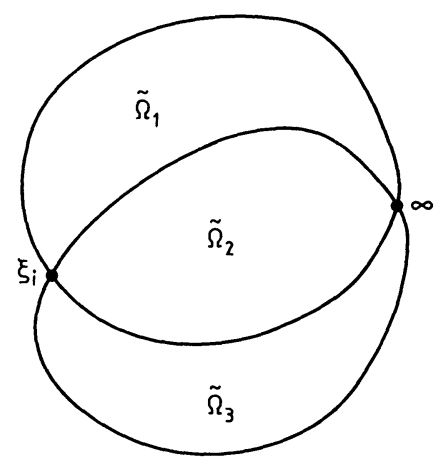

Fig. 6. Three large regions abutting infinity and a turning point $\xi_{i}$

claim that the Stokes matrix for the transition function associated with the middle region $\tilde{\Omega}_{2}$ is trivial.

Proof. The matrix $A=\alpha \sigma_{3}+\beta \sigma_{2}+\gamma \sigma_{1}$ may be diagonalized via $T^{-1} A T=\mu \sigma_{3}$, where $\mu^{2}=\alpha^{2}+\beta^{2}+\gamma^{2}$ and

$$
T=\left(\begin{array}{cc}
1 & -\frac{\gamma-i \beta}{\mu+\alpha} \\
\frac{\mu-\alpha}{\gamma-i \beta} & 1
\end{array}\right)
$$

is determined up to right-multiplication by a constant matrix. We choose the form in (A.4) so that $T \rightarrow 1$ as $\xi \rightarrow \infty$. (Moreover, $\operatorname{Diag}\left\{T^{-1} \partial T / \partial \xi\right\}=\mathcal{O}\left(\xi^{-5}\right)$.) The computation of the connection matrices $C$ thus proceeds as in [29]:

$$
C=\lim _{\xi \rightarrow \infty} \exp \left[\tau \int_{\xi_{k}}^{\xi} \mu\left(\xi^{\prime}\right) d \xi^{\prime}-\frac{\zeta^{2 l+3}}{2 l+3}-x \zeta\right] .
$$

Again following [29] we break the integral in the exponential into two pieces $I=I_{1}+I_{2}$ by integrating first to $\tilde{\xi}_{k}$ and then to infinity, where $\left|\tilde{\xi}_{k}-\xi_{k}\right| \cong \mathcal{O}\left(\tau^{-\frac{1}{2}+\varepsilon}\right)$. In $I_{2}$ we may drop the terms from $\gamma$ since these contribute $\mathcal{O}\left(\tau^{-2}\right)$ to the integrand. The resulting integral is then given by $\frac{\zeta^{2 l+3}}{2 l+3}+x \zeta+\mathcal{O}(1 / \zeta)$. The contribute from the lower limit of integration in $I_{2}$ will cancel a term from $I_{1}$. We estimate $I_{1}$ as

$$
\tau \int_{\xi_{k}}^{\tilde{\xi}_{k}} \mu\left(\xi^{\prime}\right) d \xi^{\prime}=\tau\left(\tilde{\xi}_{k}-\xi_{k}\right)^{2} \xi_{p} \dot{p}_{l}^{ \pm}\left(\xi_{k}\right) \sqrt{\xi_{k}^{2} \pm 1}+\mathcal{O}(\log \tau / \tau)
$$

where $\dot{p}$ is the derivative at the zero. Thus $C=1$ for matching in each sector.

Near a root of $p_{l}$ we expand in powers of $\xi-\xi_{k}$. Defining $\eta_{k} \equiv \tau^{1 / 2}\left(\xi-\xi_{k}\right)$ the equation takes the form

$$
\frac{d W}{d \eta_{k}}=\left[\left(A \sigma_{3}+B \sigma_{2}\right) \eta_{k}+\mathcal{O}\left(1 / \tau^{1 / 2}\right)\right] W .
$$




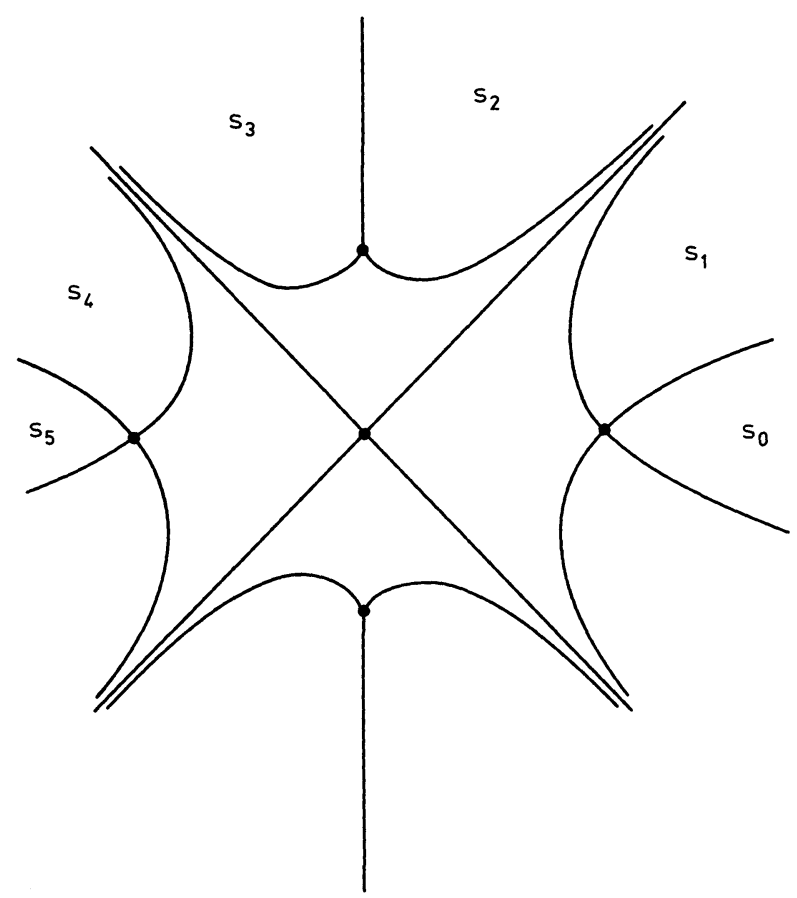

Fig. 7. Stokes lines for the case $l=1, u \sim+(-x)^{1 / 2}$ for $x \rightarrow-\infty$. We have also indicated the Stokes parameter associated with each region

This may be diagonalized by some matrix $V$ so that near the turning point we have a solution $\breve{W}_{i}$ of the equation which is asymptotic to

$$
V \exp \left[\sqrt{A^{2}+B^{2}} \sigma_{3} \frac{1}{2} \eta_{k}^{2}\right]
$$

and $\sqrt{A^{2}+B^{2}}=2 \xi_{p} \dot{p}_{l}^{ \pm}\left(\xi_{k}\right) \sqrt{\xi_{k}^{2} \pm 1}$. One may check that the limit as $\xi \rightarrow \xi_{k}$ of $T$ in the WKB ansatz exists and approaches $V$. Thus the $N$ matrices are also trivial, so that the Stokes matrix for the central region is trivial. When the regions defined by the conjugate Stokes rays emanating from a point are not of the form in Fig. 6 we cannot use the WKB ansatz to get a good approximation to one of the fundamental solutions in a sectorial domain of infinity.

We may apply this lemma to obtain the necessary conditions on the Stokes matrices associated to solutions of the string equations consistent with perturbation theory. In some cases these necessary conditions determine the Stokes parameters uniquely.

Our first task is therefore to describe the Stokes lines. In the $\varrho \rightarrow \infty$ limit there are 4 lines emerging from the origin at right angles, and at each of the points $\pm \xi_{i}$ there are four lines emerging at right angles since the zeroes of $p_{l}$ are simple. Finally at \pm 1 , or $\pm i$ there are three lines emerging at angles $2 \pi / 3$. Stokes lines can only interset at turning points. 


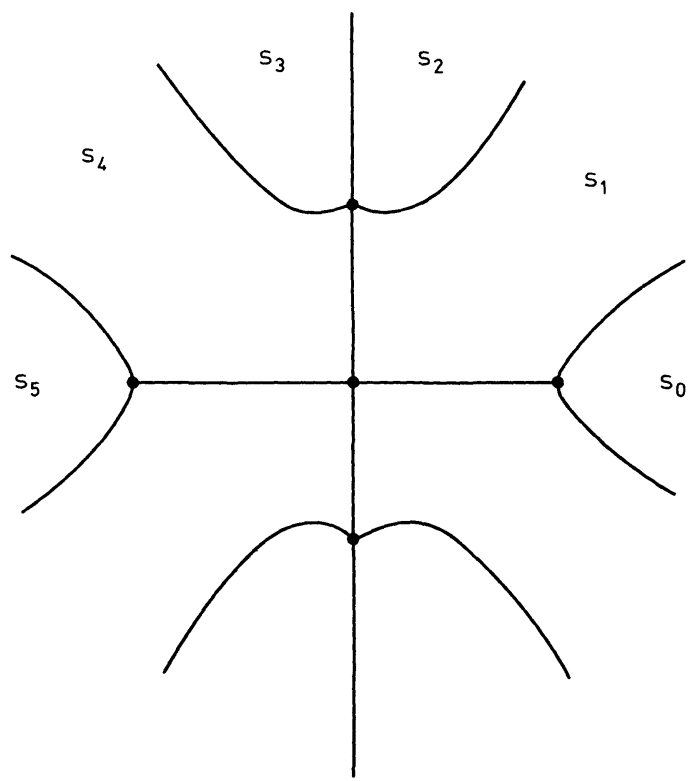

Fig. 8. Stokes lines for $l=1, u \sim-(-x)^{1 / 2}$ for $x \rightarrow-\infty$

The conjugate Stokes lines for the two cases with $l=1$ are shown in Fig. 7 and Fig. $8^{21}$. Applying the lemma and taking into account the constraints $s_{k}=$ $-\bar{s}_{k}=s_{2 l+3-k}$ and (3.13) we see that for $l=1, u \sim+(-x)^{1 / 2}, s_{5}=0$. This forces $s_{2}=s_{3}=-i$ and $s_{1}+s_{4}=s_{1}-\bar{s}_{1}=i$ but leaves the real part of $s_{1}$ undetermined. As expected, there is a one-parameter family of solutions consistent with perturbation theory. It is interesting to note that if we consider the (unphysical) case $u \sim-(-x)^{1 / 2}$ than by the lemma $s_{2}=s_{3}=0$ forcing a unique solution $s_{1}=s_{4}=s_{5}=i$, consistent with the results of [30]. Moving on the case $l=2$ we have Fig. 9 and Fig. 10. From the limit $x \rightarrow+\infty$ we obtain that $s_{1}=s_{6}=0$. From the limit $x \rightarrow-\infty$ we obtain $s_{2}=s_{5}=0$, and from the monodromy constraints we get $s_{0}=s_{3}=s_{4}=-i$, implying that there is a unique solution satisfying these constraints, as claimed. (We are assuming that the inverse monodromy problem can be solved. Strictly speaking we have only shown that if a solution exists it is completely unique. The solvability of the inverse monodromy problem could probably be demonstrated regirously using the methods in [34]. Alternatively we could use the free fermion formalism of Sect. 6 to construct a solution.)

If we move on to higher $l$ we meet a technical problem which is that the configuration of Stokes lines becomes too complicated to use our lemma immediately to set half the Stokes parameters to zero. For example, the situation for $l=4$, the next case of interest in depicted in Fig. 11 and Fig. 12. Although we may guess that, e.g., $s_{3}=0$ we cannot conclude this without further (difficult) computation. Nevertheless it is natural to suppose that for general $l$ we have the following configuration of Stokes parameters: For $l$ odd, $u \sim+(-x)^{1 /(l+1)}$

\footnotetext{
21 Although these and the following figures can be deduced by pure thought it is much faster to plot them using Mathematica
} 


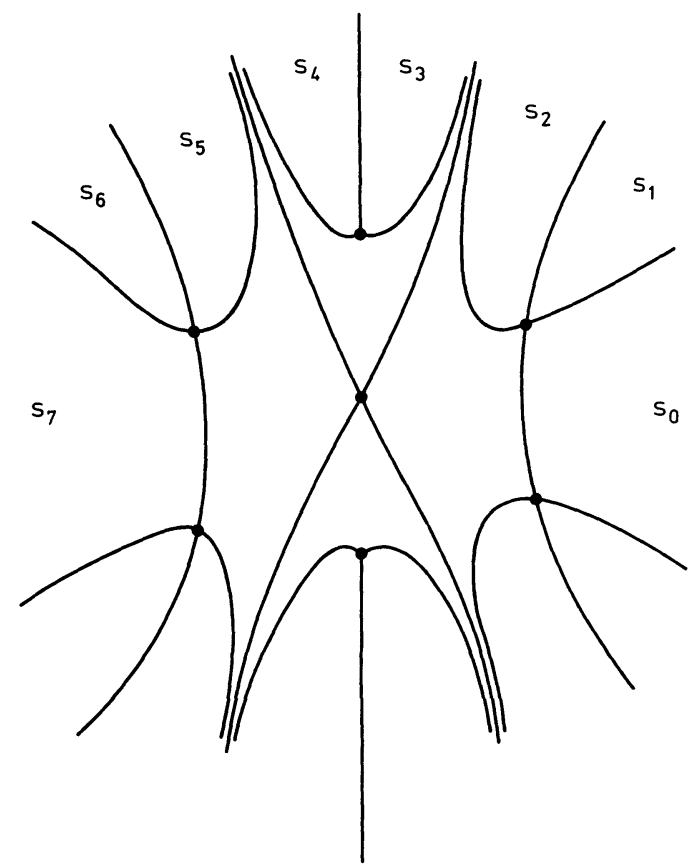

Fig. 9. Stokes lines for $l=2, u \sim x^{1 / 3}, x \rightarrow+\infty$

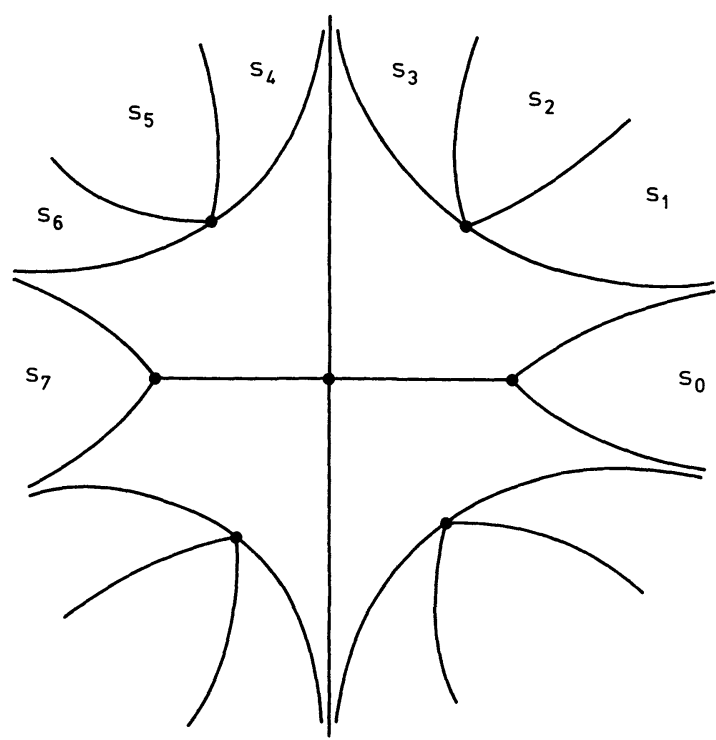

Fig. 10. Stokes lines for $l=2, u \sim x^{1 / 3}, x \rightarrow-\infty$

we have $s_{0}=s_{2}=s_{3}=\cdots=s_{l-3}=s_{l-1}=0, s_{l+1}=s_{l+2}=-i, s_{1}, s_{3}, \ldots s_{l}$ undetermined, but subject to the constraints $\alpha-\bar{\alpha}=-i$, where $\alpha=s_{1}+\cdots s_{l}$. On 


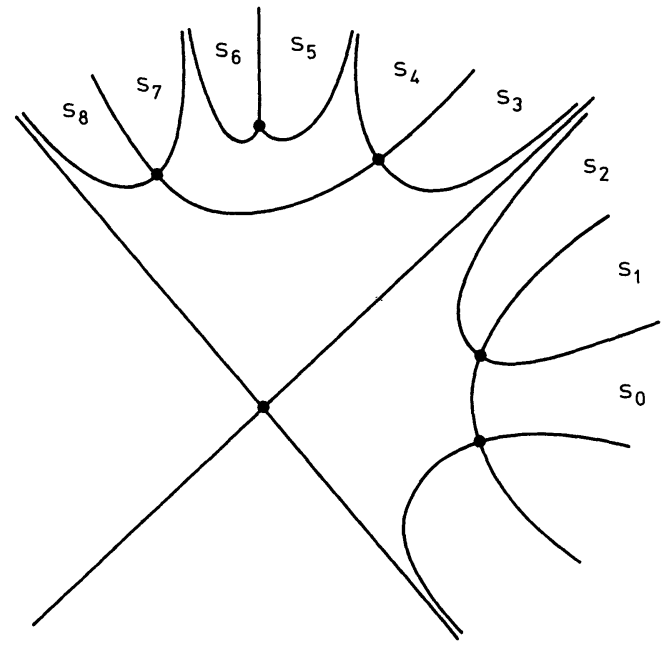

Fig. 11. Stokes lines for $l=4, u \sim x^{1 / 5}, x \rightarrow+\infty$

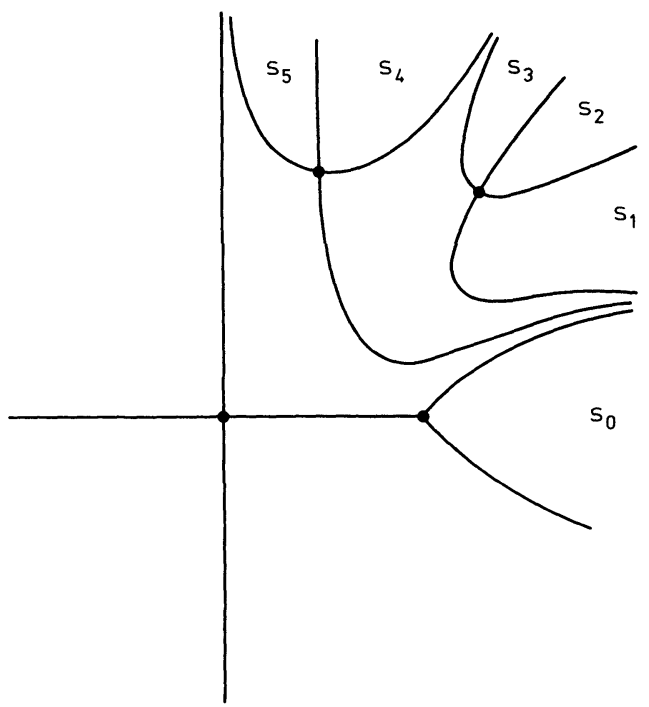

Fig. 12. Stokes lines for $l=4, u \sim x^{1 / 5}, x \rightarrow-\infty$

the other hand, for $l$ even, comparing the constraints at either end of the axis will fix two disjoint sets of Stokes parameters. We expect that $s_{1}=s_{2}=\cdots=s_{l}=0$ while $s_{l+1}=s_{l+2}=s_{2 l+3}=-i$ so that the solution is unique.

Finally we remark that we have not used the reality constraints in a very essential way. An interesting solution of the Painleve equation is the triply truncated solution, characterized by $u \sim+(-x)^{1 / 2}$ for $x \rightarrow-\infty$ and $u \sim \pm i x^{1 / 2}$ for $x \rightarrow \infty$. Using the above technique one easily shows that in this case the constraints $s_{2}=s_{3}=-i, s_{0}=s_{5}=0, s_{1}+s_{4}=0$ are supplemented by $s_{1}=0$ or 
$s_{4}=0$, depending on the sign of the imaginary part. In either case we confirm that the solution is unique.

We can use the above configurations of Stokes data and the fact that KdV flow is isomonodromic to argue that it is impossible for a solution with $l$ even to flow to a solution with $l$ odd and $u \sim+(-x)^{1 /(l+1)}$. For simplicity let us consider the string equation

$$
\frac{1}{2}\left(m_{1}+\frac{1}{2}\right) R_{m_{1}}+\frac{1}{2}\left(m_{2}+\frac{1}{2}\right) T R_{m_{2}}=x
$$

with $m_{1}>m_{2}$. As discussed in [6,63] if one scales a solution $u(x ; T)$ to (A.6) using $v(y ; T)=T^{-2 /\left(2 m_{2}+1\right)} u\left(T^{-1\left(2 m_{2}+1\right)} y ; T\right)$, then the large $T$ limit $v(y)=\lim _{T \rightarrow \infty} v(y ; T)$ must be a solution of the lower order string equation $\frac{1}{2}\left(m_{2}+\frac{1}{2}\right) R_{m_{2}}[v] \sim x$, provided the limit exists. However, the existence of this limit is a very delicate issue. Indeed, in [61] convincing evidence is presented that the flow from $m=3$ to $m=2$ does not exist. If the limit does exist then we can scale $\zeta \rightarrow T^{-1 /\left(2 m_{2}+1\right)} \zeta$ in (3.4) to obtain a connection with a smooth $T \rightarrow \infty$ limit. Since solutions can in principle be obtained from the path-ordered exponential of the connection, solutions to (3.4) will also have smooth $T \rightarrow \infty$ limits. Moreover, from the asymptotics in $\zeta$ we see that the coefficients have smooth $T \rightarrow \infty$ limits and in fact approach the asymptotics of the lower order equation. Thus, fundamental solutions smoothly approach fundamental solutions for the lower order equation, although they will be defined on small regions of angular width $\frac{2 \pi}{4 m_{1}+2}$, and hence only define part of a fundamental solution for the lower order equation which is defined on the larger regions of angular width $\frac{2 \pi}{4 m_{2}+2}$. Because of this we find two rules governing flows:

1. A large region associated with a trivial Stokes matrix for the " $m_{2}$ equation" cannot contain a small region with a nontrivial Stokes matrix for the " $m_{1}$ equation."

2. A large region associated with a nontrivial Stokes matrix for the " $m_{2}$ equation" must contain at least one small region with a nontrivial Stokes matrix for the " $m_{1}$ equation."

Note that for even and odd $l$ the nontrivial Stokes data disagrees on the real axis. Hence, by rule 1 it is impossible to use $\mathrm{KdV}$ flow to go from an even $l$ to an odd $l$ model, confirming the result of [61]. Note that flow from an even $l$ to a smaller even $l$ is consistent with rules 1 and 2 .

The present formalism might also be of use in setting the issue of whether the unique solutions for $l$ even with perturbative asymptotics can have poles on the real axis. Because of the very sparse Stokes data we can formulate the solution to (3.4) in terms of a Riemann-Hilbert problem, which further simplifies in terms of $\Psi(\lambda)$ defined in (3.3). Since an analogous RH problem for the PII equation can be used to establish the existence of pole-free solutions we suspect that the same methods can be brought to bear here. We hope to return to this issue in the future.

Unitary Matrix Models. An analog of the BMP solutions exists for the PII hierarchy [21, 64]. We may again apply our lemma for the first two cases in the series. In this case we require power law growth at one end of the axis and exponential decay at the other end. In the first two cases in the PII hierarchy 
these two constraints lead to a configuration of Stokes lines from which we may conclude necessary conditions that, together with the monodromy constraints only determine the Stokes parameters up to a single real parameter. The nontrivial Stokes matrices are again concentrated on the $y$-axis, allowing us to formulate a Riemann-Hilbert problem as in [29], Chap.4. Again, we can only conjecture that this situation continues for all the other members in the hierarchy. The one nontrivial parameters enters the integral equations equivalent to the $\mathrm{RH}$ problem. In fact the integral equations can be examined directly, and in [21] it is shown that a physically acceptable solution indeed exists for a unique value of this parameter.

\section{Appendix B. Twistor Correspondence}

Mason and Sparling [65] have shown that solutions to the $\mathrm{KdV}$ equations are in one-one correspondence with holomorphic vector bundles over minitwistor space $\left(=T \mathbb{P}^{1}\right)$ which posess an extra symmetry. Since solutions to the string equations define, in particular, solutions to the $\mathrm{KdV}$ equations we may apply the observation of [65] to associate corresponding holomorphic vector bundles over $T \mathbb{P}^{1}$.

Consider the equations:

$$
\begin{aligned}
\mathscr{L} \Psi\left(\lambda, x, T_{1}\right) & =0, \\
\left(-2 \frac{\partial}{\partial T_{1}}+\mathscr{P}_{1}\right) \Psi\left(\lambda, x, T_{j}\right) & =0
\end{aligned}
$$

which are compatible when $u\left(x, T_{1}\right)$ satisfies the $\mathrm{KdV}$ equation. By taking a linear combination of these conditions and changing the framing by

$$
\Psi \rightarrow\left(\begin{array}{cc}
1 & 0 \\
H & 1
\end{array}\right) \Psi,
$$

where $H^{\prime}=u$ we obtain the linear system which can be regarded as the twice dimensionally-reduced system equivalent to the SDYM's equations in a space of signature $(2,2)[65]$. This is similar to the relation to inverse scattering theory pointed out in [66]. Using standard twistor methods these can be regarded as defining a holomorphic structure on a two-dimensional complex vector bundle over $T \mathbb{P}^{1}$. (In brief, pulling back the connection to $\mathbb{P}^{3}(\mathbb{C})-\mathbb{P}(\mathbb{C})$ via the standard twistor fibration, the self-duality condition becomes an integrability condition for a holomorphic 2-plane bundle, and dividing out by the lift of the symmetry $\mathbb{R}^{4} \rightarrow \mathbb{R}^{4} / \mathbb{R}=\mathbb{R}^{3}$ brings us down to minitwistor space $T \mathbb{P}^{1}$. See [67] for details.) In this paper a crusial role was played by the "equation in $\lambda$," $\mathbb{P} \Psi=0$. Such equations arise naturally in the twistor construction-essentially they embody the statement that the bundle on twistor space is the pullback of a bundle on spacetime.

Acknowledgements. I am very grateful to T.Banks, E. Brézin, C.Crnkovic, M. Douglas, V. Drinfeld, I. Frenkel, H. Garland, A. Its, M. Jimbo, V. Korepin, A. Morozov, D. Pickrell, D. Sattinger, N. Seiberg, R. Shankar, S. Shatashvili, S. Shenker, and G. Zuckerman for helpful discussions. Short but influential discussions with D. Hain, A.Its, and L. Takhtadjan were useful in crystallizing the ideas about quantum Riemann surfaces. I thank N. Seiberg for very helpful comments on a preliminary version of Appendix A. I would also like to thank the theory group at Rutgers University for their hospitality while some of this work was being done. This work was supported by DOE grant DE-AC02$76 \mathrm{ER} 03075$. 


\section{References}

1. Brézin, E., Kazakov, V.: Exactly solvable field theories of closed strings. Phys. Lett. B236, 144 (1990)

2. Douglas, M., Shenker, S.: String in less than one dimension. Rutgers preprint RU-89-34

3. Gross, D., Migdal, A.: Nonperturbative two dimensional quantum gravity. Phys. Rev. Lett. 64, 127 (1990)

4. Gross, D., Migdal, A.: A nonperturbative treatment of two-dimensional quantum gravity. Princeton preprint PUPT-1159 (1989)

5. Douglas, M.: Strings in less one dimension and the generalized KdV hierarchies. Rutgers preprint RU-89-51

6. Banks, T., Douglas, M., Seiberg, N., Shenker, S.: Microscopic and macroscopic loops in nonperturbative two dimensional gravity. Rutger preprint RU-89-50

7. Witten, E.: On the structure of the topological phase of two dimensional gravity. Preprint IASSNS-HEP-89/66

8. Distler, J.: $2 D$ quantum gravity, topological field theory and multicritical matrix models. Princeton preprint PUPT-1161

9. Dijkgraaf, R., Witten, E.: Mean field theory, topological field theory, and multimatrix models. IASSNS-HEP-90/18; PUPT-1166

10. Verlinde, E., Verlinde, H.: A solution of two dimensional topological quantum gravity. Preprint IASSNS-HEP-90/40

11. Friedan, D., Shenker, S.: The analytic geometry of two-dimensional conformal field theory. Nucl. Phys. B281, 509 (1987); Friedan, D.: A new formulation of string theory. Physica Scripta T 15, 72 (1987)

12. Date, E., Jimbo, M., Kashiwara, M., Miwa, T.: Transformations groups for soliton equations. I. Proc. Jpn. Acad. 57A, 342 (1981); II. Ibid., 387; III. J. Phys. Soc. Jpn. 50, 3806 (1981); IV. Physica 4D, 343 (1982); V. Publ. RIMS, Kyoto University 18, 1111 (1982); VI. J. Phys. Soc. Jpn. 50, 3813 (1981); VII. Publ. RIMS, Kyoto University 18, 1077 (1982)

13. Segal, G., Wilson, G.: Loop groups and equations of KdV type. Publ. I.H.E.S. 61, 1 (1985)

14. Frenkel, I.: Representations of affine Lie algebras, Hecke modular forms, and Korteweg-de Vries type equations. Proceedings of the 1981 Rutgers Conference on Lie Algebras and related topics. Lecture Notes in Mathematics, vol.933, p. 71. Berlin, Heidelberg, New York: Springer 1982

15. Flaschka, H., Newell, A.: Monodromy and spectrum-preserving deformations. I. Commun. Math. Phys. 76, 65 (1980)

16. Its, A.R., Izergin, A.G., Korepin, V.E., Slavnov, N.A.: Differential equations for quantum correlation functions. Australian National University preprint; Trieste preprints, IC/89/120,107,139

17. Martinec, E.: Private communication

18. Drinfeld, Sokolov: Equations of Korteweg-de Vries type and simple Lie algebras. Sov. J. Math. 1975 (1985)

19. Gelfand, I.M., Dickii, L.A.: Asymptotic behavior of the resolvent of Sturm-Liouville equations and the algebra of the Korteweg-De Vries equations. Russian Math. Surv. 30, 77 (1975)

20. Periwal, V., Shevitz, D.: Unitary-matrix models as exactly solvable string theories. Phys. Rev. Lett. 64, 1326 (1990)

21. Crnkovic, C., Douglas, M., Moore, G.: To appear

22. Kutasov, D., Di Francesco. Ph.: Unitary minimal models coupled to $2 D$ quantum gravity. Princeton preprint, PUPT-1173

23. Witten, E.: Quantum field theory and the Jones polynomial. Commun. Math. Phys. 121, 351 (1989)

24. Ginsparg, P., Goulian, M., Plesser, M.R., Zinn-Justin, J.: $(p, q)$ string actions. Harvard preprint HUTP-90/A015;SPhT/90-049

25. Jimbo, M., Miwa, T., Ueno, K.: Monodromy preserving deformation of linear ordinary differential equations with radional coefficients. Physica 2D, 306 (1981)

26. Jimbo, M., Miwa, T.: Monodromy preserving deformation of linear ordinary differential equations with radional coefficients. II. Physica 2D, 407 (1981)

27. Sato, M., Miwa, T., Jimbo, M.: Aspects of holonomic quantum fields isomonodromic deformation and Ising model. In: Complex Analysis, Microlocal Calculus and Relativistic Quantum Theorey. Iagolnitzer, D. (ed.). Lecture Notes in Physics, vol. 126. Berlin, Heidelberg, New York: Springer 
28. Jimbo, M.: Introduction to holonomic quantum fields for mathematicians. Proc. Symp. Pure Math. 49, part I. 379 (1989)

29. Its, A., Novokshenov, V.Yu.: The isomonodromic deformation method in the theory of Painlevé equations. Lecture Notes Mathematics, vol. 1191. Berlin, Heidelberg, New York: Springer

30. Kapaev, A.: Asymptotics of solutions of the Painleve equation of the first kind. Differential Equations 24, 1107 (1988)

31. Miwa, T.: Painlevé property of monodromy preserving deformation equations and the analytic of $\tau$ functions. Publ. Res. Inst. Math. Sci. 17, 703 (1981)

32. Brezin, E., Marinari, E., Parisi, G.: A non-perturbative ambiguity free solution of a string model. Preprint ROM2F-90-09

33. David, F.: Loop equations and non-perturbative effects in two-dimensional quantum gravity. Preprint SPhT/90-043

34. Its, A.R., Novokshenov, V.Yu.: Effective sufficient conditions for the solvability of the inverse problem of monodromy theory for systems of linear ordinary differential equations. Funct. Anal. Appl. 22, 190 (1988)

35. Wasow, W.: Asymptotic expansions for ordinary differential equations. New York: Interscience 1965

36. Sibuya, Y.: Stokes phenomena. Bull. Am. Math. Soc. 83, 1075 (1977)

37. Malgrange, B.: La classification des connexions irréguliers á une variable. In: Mathématique et Physique: Sém École Norm. Sup. 1979-1982. Basel: Birkhäuser 1983

38. Jurkat, W.: Meromorphe Differentialgleichungen. Lecture Notes in Mathematics, vol. 637. Berlin, Heidelberg, New York: Springer

39. Babbitt, D., Varadarajan, V.: Local moduli for meromorphic differential equations. Bull. Am. Math. Soc. 72, 95 (1985)

40. Babitt, D., Varadarajan, V.: Deformations of nilpotent matrices over rings and reduction of analytic families of meromorphic differential equations. Mem. Am. Math. Soc. 55, 1 (1985)

41. Varadarajan, V.: Recent progress in differential equations in the complex domain. Preprint

42. Majima, J.: Asymptotic analysis for integrable connections with irregular singular points. Lecture Notes in Mathematics, vol. 1075. Berlin, Heidelberg, New York: Springer

43. Dubrovin, Matveev, Novikov: Non-linear equations of Korteweg-De Vries type, finite-zone linear operators, and abelian varieties. Russ. Math. Surveys 31, 59 (1976)

44. Mumford, D.: An algebro-geometric construction of commuting operators and of solutions to the Toda lattice equation, Korteweg-de Vries equation and related non-linear equations. Proceedings of symposium on algebraic geometry. Nagata, M. (ed.) Kinokuniya, Tokyo, 1978

45. Abramowitz, M., Stegun, I.: Handbook of mathematical functions. Dover

46. Sato, M., Miwa, T., Jimbo, M.: Holonomic quantum field theory. II. Publ. RIMS 15, 201 (1979)

47. Miwa, T.: Clifford operators and Riemann's monodromy problem. Publ. Res. Inst. Math. Sci. 17, 665 (1981)

48. Zamalodchikov, Al.B.: Conformal scalar field on the hyperelliptic curve and critical Ashkin-Teller multipoint correlation functions. Nucl. Phys. B285, 481 (1987)

49. Bershadsky, M., Radul, A.: Conformal field theories with additional $Z_{N}$ symmetry. Int. J. Mod. Phys. A2, 165 (1987)

50. Dixon, L., Friedan, D., Martinec, E., Shenker, S.: The conformal field theory of Orbifolds. Nucl. Phys. B282, 13 (1987)

51. Hamidi, S., Vafa, C.: Interactions on Orbifolds. Nucl. Phys. B279, 465 (1987)

52. Barouch, E., McCoy, B.M., Wu, T.T.: Phys. Rev. Lett. 31, 1409 (1973); Wu, T.T., McCoy, B.M., Tracy, C.A., Barouch, E.: Phys. Rev. B13, 316 (1976)

53. Witten, E.: Conformal field theory, Grassmanians, and algebraic curves. Commun. Math. Phys. 113, 189 (1988)

54. Palmer, J.: Determinants of Cauchy-Riemann operators as $\tau$-functions. Univ. of Arizona preprint; The tau function for Cauchy-Riemann operators on $S^{2}$. Unpublished letter to C. Tracey

55. Mehta, M.L.: Random matrices. New York: Academic Press 1967

56. See Sect.10.3 in Itzykson, C. and Drouffe, J.-M.: Statistical field theory, vol.2. Cambridge: Cambridge Univ. Press 1989

57. Jimbo, M., Miwa, T., Mori, Y., Sato, M.: Density matrix of an impenetrable bose gas and the fifth Painlevé transcendent. Physica 1D, 80 (1980)

58. Morozov, A., Shatashvili, S.: Private communications, Nov. 1989, and Dec. 1989

59. Moore, G., Seiberg, N.: Lectures of RCFT. Preprint RU-89-32; YCTP-P13-89 
60. Brezin, E., Marinari, E., Parisi, G.: A non-perturbative ambiguity free solution of a string model. ROM2F-90-09

61. Douglas, M., Seiberg, N., Shenker, S.: Flow and instability in quantum gravity. Rutgers preprint, RU-90-19

62. See reference [29], especially, Chap. 5

63. Crnkovic, C., Ginsparg, P., Moore, G.: The Ising model, the Yang-Lee edge singularity, and 2D quantum gravity. Phys. Lett. 237B, 196 (1990)

64. Hastings, S.P., McLeod, J.B.: A boundary value problem associated with the second painlevé transcendent and the Korteweg-de Vries equation. Arch. Rat. Mech. Anal. 73, 31 (1980)

65. Mason, Sparling: Nonlinear Schrödinger and Korteweg-De Vries are reductions of self-dual Yang-Mills. Phys. Lett. 137A, 29 (1989)

66. Belavin, A., Zakharov, V.: Yang-Mills equations as inverse scattering problem. Phys. Lett. 73B, 53 (1978)

67. Atiyah, M.F.: Collected works, vol.5. Oxford: Clarendon press 1988

Communicated by N. Yu. Reshetikhin 